\title{
ESTUDIO DE UNA EXPERIENCIA INTERNACIONAL DE APRENDIZAJE INTERACTIVO EN LA ASIGNATURA PEQUEÑAS EMPRESAS E INICIATIVA EMPRESARIAL
}

\author{
STUDY OF AN INTERNATIONAL INTERACTIVE LEARNING \\ EXPERIENCE IN THE SUBJECT SMALL BUSINESS AND \\ ENTREPRENEURSHIP
}

Dr. José María Millán Tapia

Ana Millán Tapia

Concepción Román Díaz

\begin{abstract}
Resumen
En este trabajo se describe y evalúa una experiencia internacional de aprendizaje interactivo desarrollada en la asignatura Pequeñas Empresas e Iniciativa Empresarial, perteneciente al programa Máster en Iniciativa Empresarial, Estrategia y Economía de las Organizaciones, ofertado en la Facultad de Economía de la Universidad Erasmus de Rotterdam. A través de las actividades realizadas por los estudiantes durante el desarrollo de esta asignatura (cumplimiento de un temario de tipo interdisciplinar, realización de trabajos en grupos formados por estudiantes de
\end{abstract}


varias nacionalidades, reuniones de supervisión y seguimiento con los profesores, presentación de estos trabajos en público, visita a un centro de investigación, charlas con verdaderos emprendedores y análisis de una empresa real), y por medio de un cuestionario, se analiza la percepción de los beneficios pedagógicos por parte de los estudiantes, así como su satisfacción con la metodología de enseñanza-aprendizaje utilizada, con el sistema de evaluación y con la asignatura en sí misma. La opinión de los profesores de la asignatura sobre estos aspectos también es recogida y analizada en este trabajo a través de una entrevista semi-estructurada.

\section{Palabras Clave}

Emprendimiento, experiencia internacional, aprendizaje interactivo, aprendizaje constructivista.

\section{Abstract}

This paper describes and evaluates an international interactive learning experience carried out in the Seminar of Small Business and Entrepreneurship, which is one of the subjects of the Master in Entrepreneurship, Strategy and Economy of Organizations offered by the Faculty of Economics at the Erasmus University in Rotterdam. By means of a questionnaire, we analyse the students' perception of the pedagogical benefits of the activities proposed (i.e., compliance with an interdisciplinary syllabus, carrying out works in groups made up of students from different nationalities, monitoring meetings with members of the teaching staff, public presentation of assignments, visit to a research centre, chats with real entrepreneurs and analysis of a real company), as well as their satisfaction with the teachinglearning methodology used, with the evaluation system and with the subject itself. The opinion of the teachers of the subject on these aspects is also collected and analyzed through a semi-structured interview.

\section{Key Words}

Entrepreneurship, international experience, interactive learning, constructivist learning. 


\section{Agradecimientos}

Quisiéramos en primer lugar agradecer a Ana Barquero (Universidad de Huelva), Fernando Millán (Universidad de Sevilla) y a los evaluadores anónimos por sus valiosos comentarios y sugerencias. También quisiéramos expresar nuestra gratitud a nuestros compañeros en esta experiencia docente, Joern Block, Jolanda Hessels y Peter Van der Zwan (Erasmus University Rotterdam) por su colaboración, sus consejos y sugerencias. Quisiéramos mencionar también la desinteresada ayuda de María José Ruiz (Universidad de Castilla La Mancha) en el diseño del cuestionario. Nos gustaría a su vez destacar la total disponibilidad a participar en este proyecto de los alumnos de la asignatura Pequeñas Empresas e Iniciativa Empresarial. Por último, quisiéramos agradecer la invitación de Roy Thurik (coordinador del Máster en Iniciativa Empresarial, Estrategia y Economía de las Organizaciones de la Erasmus University Rotterdam) a realizar una estancia de investigación posdoctoral en la Erasmus University Rotterdam, y a participar como docentes en esta experiencia. A todos ellos, muchas gracias.

Durante la realización de este trabajo, José María Millán contó con financiación del Ministerio de Educación (Ayudas para estancias de movilidad en el extranjero «José Castillejo» para jóvenes doctores, Programa Nacional de Movilidad de Recursos Humanos, Plan Nacional de I+D+i 2008-2011). 


\section{Introducción}

Dime y olvidaré.

Muéstrame y podría recordar.

Involúcrame y entenderé.

Confucio. Siglo V a.C.

Las lecciones magistrales pierden peso frente al trabajo personal de los alumnos y las discusiones en el aula. El Espacio Europeo de Educación Superior (EEES) perfila un modelo de aprendizaje centrado en el alumno, en el que el profesor es un facilitador del aprendizaje, el alumno tiene una autonomía creciente, la enseñanza presencial abre cada vez más paso a la virtual, a la investigación del alumno y a la construcción de su propio aprendizaje, y en ese proceso, las tecnologías de la información y la comunicación tienen un papel relevante.

Así, el objetivo fundamental de este nuevo marco educativo (en la medida en que aceptemos el argumento "utilitarista" de que la educación debe preparar a los estudiantes para la vida profesional; véase King \& Behnke, 2005) pasa por el desarrollo de atributos (conocimientos, habilidades, actitudes, valores...) que permitan enfrentarse con éxito a situaciones complejas, o en otras palabras, por el desarrollo de competencias (Lasnier, 2000). No en vano, en el mundo empresarial actual, cada vez es más importante el desarrollo de habilidades no solo técnicas, sino también sociales que permitan trabajar en equipo con éxito, esto es, cumpliendo objetivos en tiempo y forma, y manteniendo al equipo motivado y cohesionado.

Esto ha hecho que el sistema educativo universitario en general, y las escuelas de negocio en particular, caminen desde entornos de aprendizaje instructivistas hacia otros entornos de tipo constructivista (Jonassen, 1991; Kafai \& Resnick, 1996). Se evoluciona, por tanto, desde un modelo organizativo del proceso de enseñanzaaprendizaje basado en la clase teórica a otro donde se incluyen modalidades organizativas basadas en seminarios, tutorías o trabajo en equipo. Se incorporan métodos didácticos basados en el estudio de casos, el aprendizaje basado en problemas, el aprendizaje orientado a proyectos y el aprendizaje cooperativo e interactivo (Brady, 1985). Y todo ello en un entorno de creciente internacionalización de la educación que genera indudables beneficios (Hénard, Diamond \& Roseveare, 2012). 
Sin embargo, lo que es todavía un objetivo a alcanzar en ciertos países europeos, como es el caso de España (donde el impulso de ciertos sectores del sistema universitario, incluida su dirección, todavía coexiste con el rechazo de un gran número de detractores), lleva años implantado en otras regiones con un considerable éxito, como es el caso holandés. ${ }^{1}$ Pero, ¿cuáles son los factores determinantes de este éxito? ¿De verdad existen tantas diferencias entre los sistemas de aprendizaje? Y asumiendo su existencia, ¿no sería posible reproducir este sistema o al menos algunas de sus características más relevantes?

Podría argumentarse que las diferencias se están acortando (o prácticamente no existen) pues las metodologías que potencian aprendizajes basados en la resolución de problemas, el trabajo en equipo y las tutorías no suponen novedad alguna en las aulas españolas, y son previas al proceso de convergencia europea marcado por el nuevo EEES. Sin embargo, salvo honrosas excepciones, el diseño de estas actividades se sustenta sobre graves carencias, como la escasa (o nula) atención tanto al proceso de composición de grupos como a su tamaño, y la focalización de la atención en los resultados finales (habitualmente un documento de texto y/o una presentación en público), y por consiguiente la falta de una evaluación continua que supervise, recomiende y oriente al alumno en la realización del trabajo, y que evite tanto la concentración del trabajo en momentos próximos a la fecha de entrega, como las estrategias "parceladoras" que dividen la tarea global en compartimentos estancos y que se afrontan de manera individual, sin que exista un verdadero trabajo cooperativo e interactivo entre miembros (García, 2009). Es frecuente además, que en estos contextos de trabajo en equipo con poca o nula supervisión, emerjan comportamientos no deseados, tales como el "problema del polizón" ${ }^{2}$ o el "heroísmo", ${ }^{3}$ que precisamente fomenten un rechazo al trabajo en equipo, tanto por parte de algunos estudiantes, como por parte de los docentes a la hora de considerar una u otra modalidad organizativa del proceso de enseñanza-aprendizaje.

En línea con la discusión anterior, el objetivo de este trabajo es precisamente describir y evaluar una experiencia internacional de aprendizaje interactivo desarrollada en la asignatura Pequeñas Empresas e Iniciativa Empresarial, perteneciente al programa Máster en Iniciativa Empresarial, Estrategia y Economía de las Organizaciones, ofertado en la Facultad de Economía de la Universidad Erasmus de Rotterdam. Este estudio, pese a identificar algunos aspectos verdaderamente positivos de esta experiencia, sin embargo, no aspira a convertirse en un "manual de buenas 
prácticas", sino que tan solo pretende contribuir a la generación del necesario debate que debe acompañar a este proceso de implantación y consolidación del nuevo marco del EEES.

Así, a través de las actividades realizadas por los estudiantes durante el desarrollo de esta asignatura (cumplimiento de un temario de tipo interdisciplinar, realización de trabajos en grupos formados por estudiantes de varias nacionalidades, reuniones de supervisión y seguimiento con los profesores, también estos de diferentes nacionalidades, presentación de tales trabajos en público, visita a un centro de investigación, charlas con verdaderos emprendedores y análisis de una empresa real), y por medio de un cuestionario, se analiza la percepción de los beneficios pedagógicos por parte de los estudiantes, así como su satisfacción con la metodología de enseñanza-aprendizaje utilizada, con el sistema de evaluación y con la asignatura en sí misma. La opinión de los profesores de la asignatura sobre estos aspectos también es recogida y analizada en este trabajo a través de una entrevista semi-estructurada.

El presente trabajo incluye 5 secciones, de las cuales la primera es esta introducción. La sección 2 resume la experiencia de enseñanza-aprendizaje objeto de análisis, mientras que la sección 3 describe el diseño de la investigación. La sección 4 presenta y discute los resultados obtenidos y, finalmente, la sección 5 aporta las conclusiones.

\section{Descripción de la experiencia de enseñanza-aprendizaje}

Este trabajo describe y evalúa una experiencia de aprendizaje interactivo desarrollada para la asignatura Pequeñas Empresas e Iniciativa Empresarial perteneciente a una de las especializaciones o segundos ciclos de los estudios en Economía y Empresa. En particular, la asignatura se imparte dentro del programa Máster en Iniciativa Empresarial, Estrategia y Economía de las Organizaciones, ofertado por el Departamento de Economía Aplicada de la Facultad de Economía de la Universidad Erasmus de Rotterdam durante el curso académico 2010-11. ${ }^{4}$

\subsection{Contextualizando la experiencia}

Contando con raíces que se remontan a 1913 y establecida en su forma actual en 1973, la Universidad Erasmus de Rotterdam basa su oferta educativa en tres ámbitos, y en todos ellos el centro goza de reconocimiento internacional. ${ }^{5}$ Esos 
sectores son Economía y Gestión; Medicina y Salud; Derecho, Cultura y Sociedad. Cada año, unos 14.000 estudiantes se forman en sus aulas, de los cuales unos 4.300 lo hacen en la Facultad de Economía. Esta facultad posee una marcada dimensión internacional y es una institución con una clara vocación de excelencia. ${ }^{6}$

El Máster en Iniciativa Empresarial, Estrategia y Economía de las Organizaciones tiene una carga lectiva de 60 créditos ECTS y un año académico de duración. Su objetivo es formar a futuros consultores, managers o emprendedores para que puedan desenvolverse nacional e internacionalmente en el ámbito del sector privado más profesionalizado. Con una fuerte orientación internacional, el máster se imparte íntegramente en inglés por docentes tanto holandeses como extranjeros. Dentro de este máster, y con un peso de 12 créditos ECTS, se encuentra la asignatura Pequeñas Empresas e Iniciativa Empresarial, cuyo desarrollo analizamos en este trabajo.

\subsection{Alumnado}

Un total de 24 estudiantes han formado el grupo de discentes en esta asignatura, que estaba compuesto por jóvenes entre 21 y 27 años (10 son mujeres y 14 varones). Como acostumbra a ocurrir en las aulas de esta universidad, el grupo ha sido de carácter eminentemente internacional, con tan solo 11 holandeses frente a 13 extranjeros. Sus países de procedencia varían desde los más cercanos como Alemania (3), Suiza (1), Grecia (3), y Bulgaria (1), hasta los más alejados como Letonia (1), Rusia (1), Brasil (1) o Costa Rica (1). Estos datos están en consonancia con la duplicación que ha experimentado en el periodo 2000-10 el número de estudiantes universitarios que realizan sus ciclos formativos en países diferentes al propio (OCDE, 2012).

La mayoría de estos estudiantes obtuvo el título de Grado en su país de procedencia, con la excepción del estudiante búlgaro y los 3 estudiantes alemanes, que cursaron sus estudios en Holanda. ${ }^{8}$ En total, 14 instituciones diferentes están involucradas en la obtención del título de Grado de estos estudiantes, de las cuales 6 son holandesas. ${ }^{9}$ En relación al tipo de estudios, la gran mayoría (20) son estudiantes que obtuvieron el Grado en Economía, mientras que 3 estudiantes lo hicieron en Administración y Dirección de Empresas, y tan solo uno de ellos en Finanzas.

Para conocer su competencia como discentes, en el marco del cuestionario anónimo que sirve de base para este trabajo, los estudiantes fueron preguntados 
acerca de sus calificaciones durante sus estudios previos de Grado. En particular, estábamos interesados en saber si sus calificaciones estaban en la media de sus compañeros de clase, por encima o por debajo. En este sentido, tan solo un estudiante declaró que sus calificaciones de Grado estuvieron por debajo de la media, mientras que 9 estudiantes afirmaron haber estado por encima de la media. El resto (14) se clasificó como estudiante de tipo medio, en relación con sus calificaciones.

En lo relativo a su experiencia laboral previa, ninguno de los 24 estudiantes matriculados en esta asignatura carecía de experiencia laboral en el momento de cumplimentar el cuestionario. Así, 21 estudiantes declararon tener experiencia como empleados asalariados a tiempo parcial, y 10 declararon tener esa experiencia como trabajadores a tiempo completo. Además, 4 estudiantes afirmaron haber trabajado en empresas familiares a tiempo parcial. Por último, 2 estudiantes declararon tener experiencia trabajando por cuenta propia a tiempo parcial, mientras que 5 declararon haber sido emprendedores a tiempo completo. ${ }^{10}$

\subsection{Profesorado}

La educación superior contemporánea debe sustentarse en colaboraciones y alianzas entre académicos e investigadores de diferentes países. ${ }^{11}$ Sobre la base de este principio, también el grupo de docentes ha tenido un marcado carácter internacional. Así, entre los 5 profesores de esta asignatura encontrábamos tres nacionalidades diferentes ( 2 holandeses, un alemán y dos españoles). Sus edades variaban entre los 26 y los 33 años, siendo 2 de ellos mujeres y 3 varones. En cuanto a su formación académica, cuatro de ellos eran Doctores en Economía mientras que uno defendió su tesis doctoral en mayo de ese mismo año 2011, pocos meses después de impartir esta asignatura. ${ }^{12}$ Su experiencia docente variaba entre los 3 años del candidato a Doctor y los 10 años de uno de los profesores españoles. Todos ellos tienen líneas de investigación similares y compatibles con la supervisión de la asignatura. $^{13}$

\subsection{Objetivos y características generales de la asignatura}

El objetivo fundamental de la asignatura Pequeñas Empresas e Iniciativa Empresarial es que los estudiantes apliquen sus conocimientos académicos y las 
herramientas del método científico a los problemas a que se enfrentan tanto gerentes como emprendedores en la empresa moderna.

En particular, la asignatura se centra en el análisis de tres áreas fundamentales para la actividad empresarial: (i) Iniciativa Empresarial; (ii) Estrategia Empresarial; y (iii) Valoración Empresarial. Así, la asignatura emplaza a los estudiantes a que trabajen en grupo y apliquen sus conocimientos académicos para escribir y defender varios trabajos sobre estas tres áreas de interés, en consonancia con las herramientas del método científico y bajo la tutorización y seguimiento de los profesores.

En este sentido, la asignatura es eminentemente multidisciplinar, teniendo los estudiantes que hacer uso de sus conocimientos en Economía, Econometría, Estrategia y Finanzas, y ampliarlos cuando así sea necesario, recurriendo a las fuentes de información que consideren oportunas, y bajo el amparo de las nuevas tecnologías.

La metodología de enseñanza-aprendizaje es constructivista, y se diseña como una "clase invertida" (inverted classroom, flipped classroom, o flip teaching; Lage, Platt \& Treglia, 2000) que, a la luz de la literatura existente, genera mejoras notables en los procesos de aprendizaje y los resultados académicos (Crouch \& Mazur, 2001; Fagen, Crouch \& Mazur, 2002). Tal como indica su nombre, en la "clase invertida" se invierte el orden establecido en el aprendizaje tradicional: la adquisición de la información la realiza el alumno fuera del aula, y el tiempo en el aula con el profesor, que es el más valioso porque es muy limitado, se dedica a que haya una verdadera interacción entre el profesor y los alumnos con el fin de ayudar a la asimilación de la información. De esta forma, el profesor puede poner en valor su papel en el proceso de enseñanzaaprendizaje con diferentes técnicas que fomenten el aprendizaje activo de los alumnos, como por ejemplo la resolución de problemas con los alumnos, la realización de debates, la presentación de trabajos en clase por los alumnos, etc. (Luján-Mora, 2013).

En la "clase invertida" diseñada para esta asignatura, destacan dos recursos didácticos que se solapan y vertebran el aprendizaje: (i) el "aprendizaje basado en proyectos" (Nunes de Oliveira, 2011); y (ii) el denominado "escribir para aprender" (Emig, 1977; Román, Millán \& Millán, 2016), que considera la escritura un proceso generador de ideas y creador de conocimiento, en vez de un mero medio para transmitir información o resumir conceptos. Ambos recursos se utilizan de manera intensiva durante la realización por parte de los estudiantes de los trabajos sobre actividad empresarial descritos anteriormente. 
En este sentido, el proceso de adquisición de la información que se emplea para el aprendizaje lo realiza el alumno de forma autónoma y a su ritmo a partir de los materiales docentes recomendados por el profesor, como artículos académicos y materiales docentes. Al trabajo en el aula con el profesor, por su parte, tan solo se destinan 16 horas de los 12 créditos ECTS de que consta la asignatura. De estas 16 horas, tan solo 5 tienen el perfil de clases magistrales. El resto del trabajo en el aula se dedica en su totalidad a la exposición y discusión de los trabajos realizados por los estudiantes, así como a la resolución de dudas que afecten de manera transversal al conjunto de estudiantes. De este modo, se persigue intensificar la participación, interacción y compromiso de los estudiantes en el aula, fomentando que hagan preguntas, expresen sus dudas, compartan ideas y conocimientos y, en definitiva, facilitando la asimilación de la información.

\subsection{Descripción de las actividades realizadas}

La Tabla 1 que presentamos a continuación muestra el cronograma de actividades realizadas durante el desarrollo de la asignatura Pequeñas Empresas e Iniciativa Empresarial.

\section{Semana 1: 4 de Noviembre de 2010}

- Sesión introductoria a la asignatura

- Sesión introductoria al trabajo 1: "Trabajo de Investigación en Iniciativa Empresarial"

\section{Semana 2: 10 de Noviembre de 2010}

- Presentación de las "Propuestas de Trabajos de Investigación en Iniciativa Empresarial"

- Charla con emprendedor 1

\section{Semana 3: 17 de Noviembre de 2010}

- Reuniones de tutorización y seguimiento sobre el "Trabajo de Investigación en Iniciativa Empresarial"

Semana 4: 24 de Noviembre de 2010

- Visita al centro de investigación EIM Business and Policy Research, Zoetermeer 


\section{(Holanda)}

- Presentación de los "Trabajos de investigación en Iniciativa Empresarial"

- Premio a la mejor presentación de los "Trabajos de investigación en Iniciativa Empresarial"

\section{Semana 5: 1 de Diciembre de 2010}

- Entrega de los "Trabajos de Investigación en Iniciativa Empresarial"

- Charla con emprendedor 2

- Sesión introductoria al trabajo 2: "Trabajo sobre Estrategia Empresarial"

\section{Semana 6: 8 de Diciembre de 2010}

- Entrega y presentación de los "Trabajos sobre Estrategia Empresarial"

- Sesión introductoria al trabajo 3: "Trabajo sobre Finanzas Empresariales"

\section{Semana 7: 15 de Diciembre de 2010}

- Entrega y presentación de los "Trabajos sobre Valoración Empresarial"

- Calificaciones finales

- Cumplimentación del cuestionario

Tabla 1. Cronograma de actividades

\section{$\underline{\text { Semana } 1}$}

La asignatura comienza con una breve presentación de profesores y estudiantes. A continuación, se describe el contenido y las líneas generales de la asignatura, junto con el método de evaluación (que describiremos más adelante en esta sección) y los protocolos de entrega y recepción de trabajos (siempre en formato electrónico) y de comunicación entre profesores y estudiantes. ${ }^{14}$

A continuación, se describen las características del primer trabajo correspondiente a la asignatura. Se trata de realizar un "Trabajo de investigación en Iniciativa Empresarial', bajo la estructura de un artículo científico. Para la realización de este trabajo, se componen 6 equipos de 4 estudiantes, lo más heterogéneos posibles por género y nacionalidad, y se nombran 1 o 2 supervisores entre los profesores de la asignatura.

Este trabajo ha sido previamente diseñado por los supervisores, dentro del área de Iniciativa Empresarial, y relacionado con sus propias líneas de investigación, lo que 
permite una mejor tutorización y seguimiento del trabajo. Pese a que se buscan temáticas lo más interesantes y novedosas posibles, ${ }^{15}$ lo más relevante en su realización es contribuir a que los estudiantes adquieran destreza en la investigación bajo las herramientas del método científico y sepan plasmar los resultados obtenidos en un documento estructurado, que pueda ser presentado posteriormente.

Durante la presentación, se asesora a los estudiantes sobre cómo desarrollar su trabajo, se repasan algunas técnicas econométricas (que deben conocer previamente de sus estudios de Grado) y se describen los datos que podrán utilizar durante su análisis. Como resultado, los estudiantes deben presentar un trabajo que incluya título, resumen, introducción (motivación y objeto de la investigación), revisión de la literatura tanto teórica como empírica, establecimiento de hipótesis, descripción de datos, metodología de análisis, resumen de resultados más relevantes, conclusiones (incluyendo limitaciones y recomendaciones para futuras líneas de investigación), referencias bibliográficas y apéndices (si fueran necesarios). ${ }^{16}$

Esta presentación se sube inmediatamente a la plataforma virtual de la asignatura, junto con un documento especialmente preparado para cada grupo en el que se detalla: (i) una breve motivación de por qué el trabajo encomendado es interesante; (ii) una serie de preguntas generales que su trabajo debe contestar (que pueden ser ampliadas y modificadas si así lo consideran durante la realización del trabajo); (iii) un listado de trabajos publicados sobre el tema que los estudiantes deben consultar; (iv) un listado de revistas que tratan de forma sistemática el objeto de su investigación; y (v) unas instrucciones precisas sobre cómo deben presentar su trabajo final. A partir de este instante, serán los estudiantes los que crearán su propio conocimiento, siendo el tutor el encargado de guiar ese proceso.

\section{Semana 2}

Una semana más tarde, cada grupo debe presentar en el aula sus "Propuestas de Trabajo de Investigación en Iniciativa Empresarial' ante profesores y resto de grupos. Si bien la presentación oral en clase no es una actividad necesariamente nueva en el aula, en el caso de esta asignatura no es un recurso didáctico anecdótico sino que se repite en las semanas $2,4,6$ y 7 , y se diseña desde la perspectiva de los objetivos del EEES, esto es, el desarrollo de habilidades y competencias, incentivando la 
participación y el trabajo del estudiante, tanto dentro como fuera del aula (Torres-Pérez, 2012).

En particular, estas propuestas deben ser entregadas a través de la plataforma con un día de antelación a la sesión en el aula, en un documento de un máximo de 900 palabras. Esto permite a los profesores evaluar todas las propuestas y discutirlas entre ellos, para poder ofrecer en el aula recomendaciones y sugerencias más ajustadas a las necesidades de cada grupo de trabajo. Además, estas sugerencias y recomendaciones, junto con los errores o imprecisiones cometidos de forma sistemática por todos los grupos, son recogidas en un breve documento, que es entregado y comentado con todos los grupos tras las presentaciones previstas para el día siguiente.

En definitiva, se espera que las respectivas "Propuestas de Trabajo de Investigación en Iniciativa Empresarial' incluyan (i) un título preliminar del trabajo; (ii) las preguntas que el trabajo pretende resolver; (iii) una argumentación de por qué el trabajo es importante desde el punto de vista práctico o de la política económica; (iv) una descripción de la metodología que se pretende aplicar, justificando por qué es la más apropiada; (v) una descripción de la base de datos a utilizar y de las variables específicas que serán consideradas en el análisis; (vi) los resultados que a priori podrían obtenerse; y (vii) una breve argumentación de la relevancia de los mismos.

Estas presentaciones deben ser capaces de condensar toda esta información en unos 5-7 minutos, y son realizadas de forma coordinada por 2 miembros de cada grupo (quedando a criterio del grupo la elección de los ponentes). Los 10 minutos siguientes se dedican a la discusión de la presentación por profesores y resto de grupos. Además, para activar este debate, se emplaza a cada grupo a que prepare 3 puntos de discusión sobre su propia presentación, cubriendo aquellos puntos sobre los que podrían necesitar algunos consejos o recomendaciones.

Finalmente, tras las presentaciones y tras comentar el documento de sugerencias y recomendaciones, se cierra la sesión incluyendo la participación de un emprendedor, que realiza una presentación de su idea de negocio. ${ }^{17} \mathrm{El}$ objetivo es acercar a los estudiantes al fenómeno del emprendimiento y la iniciativa empresarial de la forma más realista y cercana posible, alejada de los fríos datos que han de utilizar en la realización de su trabajo de investigación. De este modo, el emprendedor comparte con el grupo la experiencia vivida al iniciar y desarrollar su aventura empresarial, los problemas a los que se ha enfrentado (de tipo burocrático, financiero, de gestión o de 
otra índole), los logros conseguidos y las perspectivas futuras de supervivencia y crecimiento.

\section{$\underline{\text { Semana } 3}$}

La sesión correspondiente a la tercera semana tiene como objetivo mantener una reunión de tutorización y seguimiento con cada uno de los grupos. Estas reuniones se llevan a cabo en su sentido más amplio, esto es, como verdaderas acciones de intervención formativa destinadas a la información, formación, orientación y seguimiento de los estudiantes (Arbizu, Lobato \& Del Castillo, 2005). En este sentido, los efectos positivos de estas actividades de tutorización sobre los resultados académicos y las actitudes hacia la materia objeto de estudio han sido ampliamente respaldadas por la literatura académica (Cohen, Kulik \& Kulik, 1982). En el caso particular de alumnos universitarios, estas actividades fortalecen además la adquisición de competencias y habilidades necesarias para su desarrollo personal y profesional (Arbizu et al., 2005; Cuevas-Salazar, García-López, Vales-García \& Cruz-Medina, 2017).

Estas reuniones, cuya duración aproximada es de una hora, no tienen lugar en el aula, sino que se realizan con cada grupo en los respectivos despachos de los profesores. Para el máximo aprovechamiento de la reunión, se insta a los grupos a que contacten vía e-mail con los profesores de forma previa a la reunión, anticipándoles lo que serán sus dudas y cuestiones más relevantes para poder seguir desarrollando su trabajo de investigación. Esto permite nuevamente a los docentes poder preparar la reunión, de forma que sus recomendaciones y sugerencias no se alimenten únicamente de la espontaneidad de la reunión, sino de una reflexión más profunda y sosegada, que debe conducir a un mejor ajuste a las verdaderas necesidades de cada trabajo en marcha.

\section{Semana 4}

Las excelentes relaciones existentes entre el personal del centro de investigación EIM Business and Policy Research y el Departamento de Economía Aplicada de la Universidad Erasmus de Rotterdam permiten celebrar la cuarta sesión en este centro. Con sede en los Países Bajos, el EIM es una organización independiente de investigación y consultoría que ofrece asesoramiento a gobiernos internacionales, nacionales, regionales y locales en el desarrollo y evaluación de sus políticas 
económicas y empresariales, y en otros temas como transporte y movilidad, empleo y asuntos sociales. Entre sus clientes destacan la Comisión Europea, la OCDE y el Banco Mundial. ${ }^{18}$

Tras visitar sus instalaciones, la sesión comienza con la participación de uno de los directores del centro, que realiza la primera presentación. En ella, se destacan aspectos tales como los objetivos y la importancia de la investigación que realiza el centro, y quiénes son sus clientes potenciales y reales. También se invita a los estudiantes a revisar la colección de documentos de trabajo que publica periódicamente el centro, que podría serles útil durante su formación académica. Finalmente, se insta a los estudiantes a contactar en un futuro próximo con el centro por si hubiera posibilidades reales de contratación o de realización de una tesis doctoral (sin ningún tipo de compromiso por ninguna de las partes).

A continuación, se inician las presentaciones de los "Trabajos de Investigación en Iniciativa Empresarial". Nuevamente, se realizan de forma coordinada por 2 miembros de cada grupo (quedando también a criterio del grupo la elección de los ponentes). El tiempo estimado para cada presentación se amplía a 10 minutos, coincidiendo en este caso con el tiempo destinado a comentar y discutir cada trabajo. Además de profesores y estudiantes, asisten a estas presentaciones algunos de los investigadores del centro de investigación, con el objetivo de enriquecer el contenido de los debates que se originan tras cada presentación. Nuevamente, estas presentaciones han sido enviadas con un día de antelación a los profesores a través de la plataforma para su análisis previo. Esto permite seguir guiando y tutorizando el trabajo de los grupos con las máximas garantías. Al finalizar las mismas, se pide a cada grupo que decida qué grupo debe ser galardonado, en su opinión, con el "Premio a la Mejor presentación del Trabajo de Investigación" (Best Research Paper Presentation Award). Finalmente, mientras los estudiantes disfrutan de un ágape por cortesía del centro de investigación, los profesores acuerdan el equipo ganador. ${ }^{19} \mathrm{El}$ acto se cierra anunciando el equipo ganador, previa exposición de los motivos que determinan el fallo final sobre el premio.

Sin embargo, los estudiantes aún tendrán algunos días más (hasta el día antes de la quinta sesión) para incorporar a su versión final de los "Trabajos de Investigación en Iniciativa Empresarial" las sugerencias y recomendaciones recibidas durante la presentación en el centro de investigación. El objetivo es interiorizar que cualquier trabajo de investigación (ya sea de carácter científico o de otra índole) no nace en su 
versión definitiva, sino que debe ser un documento "vivo" que requiere un meticuloso proceso de revisión, y que de su exposición en diferentes foros surgen recomendaciones y sugerencias que (si son válidas) deben ser incorporadas. De este modo, a través de este proceso permanente, los trabajos evolucionan y se van acercando paulatinamente a su versión final.

\section{Semana 5}

La quinta sesión comienza con una introducción a la "Estrategia Empresarial", que será la temática de la siguiente tarea de la asignatura. La presentación pretende repasar este concepto (que debió ser cubierto en sus estudios previos de Grado) desde un punto de vista teórico, centrándose en la importancia del desarrollo de nuevos productos, la estimación del tamaño de los mercados potenciales para estos productos y las diferentes vías de conquista de estos mercados potenciales.

A continuación participa otro emprendedor, que también realiza una presentación de su idea de negocio, como así ocurriera en la segunda semana. ${ }^{20}$ En esta ocasión, el objetivo de la presentación es doble. Por un lado, sigue vigente la idea de acercar a los estudiantes al fenómeno del emprendimiento. Sin embargo, esta iniciativa empresarial será también el particular objeto de análisis por parte de los estudiantes en los "Trabajos sobre Estrategia Empresarial", la siguiente tarea del curso. ${ }^{21}$ Para la realización de este trabajo, se componen 6 nuevos equipos de 4 estudiantes, siguiendo el criterio de heterogeneidad en género y nacionalidad.

En particular, la tarea se divide en (i) encontrar nuevas aplicaciones a la tecnología desarrollada por la empresa que permitan derivar en futuros productos comercializables, (ii) estimar el tamaño del mercado potencial de estos nuevos productos; y (iii) diseñar la estrategia de entrada en el mercado con estos nuevos productos. El objetivo es que los estudiantes, a través del análisis de un caso específico, conozcan y apliquen técnicas que favorezcan el desarrollo de la creatividad y aprendan tanto métodos de estimación de mercados potenciales como diferentes estrategias de penetración en estos mercados para empresas pequeñas e innovadoras.

Cada grupo tendrá que centrarse en una de las 3 tareas específicas, siendo un sorteo el que decida la distribución de las mismas. ${ }^{22}$ Se espera que durante esta semana cada grupo prepare tanto un documento escrito de un máximo de 1.200 palabras como una presentación de este documento (que tendrá lugar al inicio de la 
siguiente sesión). En esta ocasión, no se asignará tutor o tutores específicos para cada grupo, sino que se pretende que sea cada grupo el que decida a qué profesor 0 profesores acudir para la realización de sus consultas o para la resolución de dudas específicas. Como novedad, será también un sorteo el que elija uno de los ponentes de cada grupo, siendo tan solo el segundo elegido a criterio de los propios grupos. El objetivo es que al finalizar la asignatura el máximo número de estudiantes haya tenido la oportunidad de participar en alguna presentación.

\section{Semana 6}

La penúltima sesión comienza con las presentaciones de los "Trabajos sobre Estrategia Empresarial" (que han sido entregados a través de la plataforma con un día de antelación) ante profesores, resto de grupos y el propio emprendedor, cuya empresa ha sido objeto de estudio. Siguiendo nuestra práctica habitual, tanto las presentaciones como los correspondientes debates se extienden por espacio de unos 10 minutos. Además, las presentaciones y sus respectivos debates se utilizan para repasar los conceptos más interesantes sobre "Estrategia Empresarial".

A continuación se introduce el tópico de "Valoración Empresarial", que será desarrollado en la última tarea de la asignatura. Con esta presentación, se pretende repasar diferentes técnicas para valorar un proyecto empresarial concreto. ${ }^{23}$ Finalmente, se presenta la tarea específica sobre "Valoración Empresarial" que cada grupo tendrá que desarrollar durante la última semana (y presentar en la última sesión). En esta ocasión, se repiten tanto los grupos de estudiantes diseñados para los "Trabajos sobre Estrategia Empresarial" como la decisión de no asignar tutor o tutores específicos para cada grupo, quedando estas consultas a criterio de cada grupo. Dado que las empresas son en general reticentes a mostrar datos sobre los aspectos financieros de su negocio, se opta por el "Método del Caso" para abordar este último bloque. Se simula por tanto una empresa innovadora y de nueva creación, que se enfrenta a la toma de 3 decisiones fundamentales para el futuro de su negocio. Cada grupo tendrá que centrarse en una de las tres tareas específicas, siendo de nuevo un sorteo el que decide su distribución entre grupos. Para la toma de estas decisiones, sus hipotéticos dueños (cada grupo de estudiantes) deben ser capaces de valorar su 
negocio ante múltiples circunstancias y avatares. Para ello, tienen que hacer supuestos razonables y razonados sobre los diferentes escenarios a los que puede enfrentarse esta empresa: pesimistas, realistas u optimistas. Se pretende con ello que los estudiantes sean capaces de valorar una empresa, no solo en el momento actual, sino imaginando escenarios futuros y aplicando el método de valoración más apropiado a las circunstancias descritas.

Se espera que durante esta última semana cada grupo prepare tanto un documento escrito de un máximo de 1.800 palabras como una presentación de este documento (que tendrá lugar al inicio de la última sesión).

\section{Semana 7}

Al igual que la sesión anterior, esta última sesión comienza con las presentaciones de los "Trabajos sobre Valoración Empresarial" (entregados el día anterior, a través de la plataforma). En esta ocasión, se permite algo más de tiempo para cada presentación (unos 15 minutos). También se aprovecha esta vez para relacionar las presentaciones y sus respectivos debates con conceptos clave sobre "Valoración Empresarial". La asignatura se cierra con un breve debate entre profesores y estudiantes sobre las reflexiones y conclusiones generales que podrían extraerse a través del temario desarrollado en la asignatura.

A continuación, se interrumpe la sesión durante el tiempo necesario para que los profesores puedan deliberar e incorporar las calificaciones correspondientes a este bloque y se cita a los estudiantes dos horas más tarde para comunicarles sus calificaciones finales y para que cumplimenten el cuestionario final que sustenta el análisis de sus impresiones y opiniones en este trabajo. ${ }^{24}$

\subsection{Adquisición de competencias}

Probablemente sea ahora el momento, tras describir la organización de la asignatura de manera general y pormenorizada por semanas, cuando tenga sentido el presentar de forma (no necesariamente exhaustiva) las competencias tanto académicas como profesionales más relevantes cuya adquisición pretende fomentar esta asignatura. De este modo, será más sencillo asociar la relación existente entre 
actividades realizadas y competencias concretas desarrolladas. A continuación mostramos estas competencias:

i. Comprender y utilizar el "método científico".

ii. Llevar a la práctica los conocimientos teóricos adquiridos.

iii. Habituar al estudiante a analizar la realidad desde un enfoque multidisciplinar.

iv. Fomentar el trabajo en equipo, la colaboración, el consenso, la negociación, la resolución de conflictos y el respeto a la diversidad de opiniones en un contexto internacional.

v. Desarrollar la capacidad del estudiante para buscar información a través de distintas fuentes, así como su capacidad de análisis, de crítica y de síntesis.

vi. Habituar al estudiante a razonar en el marco de distintos supuestos.

vii. Fomentar la actitud crítica de los estudiantes

viii. Fomentar las habilidades técnicas y analíticas necesarias para la toma de decisiones económicas.

ix. Aplicar al análisis de los problemas criterios profesionales basados en el manejo de metodologías específicas.

x. Conseguir habilidades para argumentar y expresarse de forma coherente, bien sea en la elaboración de un texto escrito o en la exposición pública de los resultados de un estudio concreto.

xi. Fomentar la sensibilidad hacia temas de la realidad socio-económica y empresarial del entorno.

xii. Fomentar una actitud emprendedora y positiva frente a problemas nuevos, y el espíritu de superación ante el fracaso.

\subsection{Sistema de evaluación}

Las competencias académicas y profesionales objeto de evaluación son una amalgama compleja de aptitudes, actitudes y motivación. Por ello, el sistema de evaluación del que se infiera la consecución de esas competencias ha de ser lo suficientemente complejo y preciso para que sea realmente válido. Por ello, para evaluar competencias complejas en vez de habilidades concretas en un momento 
puntual, se deben considerar otras formas e instrumentos de evaluación distintos a los tradicionales como el portafolio, el diario valorativo, o la rúbrica (Arribas, 2012). Además, la idoneidad de los diferentes sistemas de evaluación no depende únicamente de su capacidad para poner de manifiesto unas u otras competencias sino que, es conveniente tener en cuenta, además, algunas consideraciones de orden práctico, con el objeto de que la evaluación sea un elemento eficaz y coherente con el resto de elementos del proceso de enseñanza-aprendizaje (Bolívar, 2007; Márquez et al. 2011; Arribas 2012).

Esta asignatura pretende cumplir, además de con la correspondiente función certificadora, con la función formativa que implica, necesariamente, una evaluación continua entendida no como una sucesión de pruebas aisladas e improvisadas sino como un proceso perfectamente planificado en todos sus aspectos (Cabrera, 2003; López-Pastor, 2012). Esta evaluación permite al estudiante una asimilación progresiva de los contenidos y una mayor interrelación con el profesor, posibilitando la puesta en práctica de uno de los aspectos más importantes de la evaluación formativa, el feedback, esto es, la toma de conciencia por parte del alumno de su propio aprendizaje (Nicol \& Macfarlane-Dick, 2006; Weaver, 2006; Boud, 2007).

La Tabla 2 muestra a continuación un esquema sobre los elementos que influyen en la calificación final de la asignatura y sus pesos relativos. Como veremos, si bien el método de evaluación utilizado tal vez no pueda ser considerado una rúbrica en su sentido más estricto (Marín-Díaz, Cabero-Almenara \& Barroso-Osuna, 2012), sí que ofrece un nivel de desagregación, detalle y pautas que lo aproximan a una versión más liviana de este método.

\section{Trabajos de Investigación en Iniciativa Empresarial (60\%)}

- Propuesta de "Trabajo de Investigación en Iniciativa Empresarial” y su presentación: 20\%

- “Trabajo de Investigación en Iniciativa Empresarial” y su presentación: 70\%

- Evaluación individual: 10\%

Trabajos sobre Estrategia Empresarial (20\%)

- "Trabajo sobre Estrategia Empresarial” y su presentación: 90\%

- Evaluación individual: 10\% 


\section{Trabajos sobre Valoración Empresarial (20\%)}

- "Trabajo sobre Valoración Empresarial" y su presentación: 90\%

- Evaluación individual: 10\%

Tabla 2. Sistema de evaluación

La calificación final que obtiene cada estudiante en esta asignatura surge de la evaluación de su desempeño en las tres áreas fundamentales: Investigación en Iniciativa Empresarial (60\%), Estrategia Empresarial (20\%) y Valoración Empresarial (20\%).

Estas calificaciones se deciden de forma consensuada por todos los profesores de la asignatura durante las reuniones semanales de coordinación. En estas reuniones, además de comentar la marcha general de la asignatura, se dedica un tiempo específico para comentar el trabajo de cada grupo de manera individualizada.

En lo que respecta al trabajo escrito, se intenta tener en cuenta tanto la dificultad de la tarea encomendada en sí misma como la capacidad para abordar su ejecución. En este sentido, se valoran aspectos tales como (i) el uso adecuado de diversas fuentes de información; (ii) la elección de herramientas de análisis ajustadas a la naturaleza de la tarea; (iii) la destreza a la hora de aplicar sus conocimientos académicos (iv) la adecuación a los estándares del método científico; (v) la creatividad; (vi) el esfuerzo; y por supuesto, (vii) la calidad del resultado final.

Evaluar la calidad de las presentaciones, como elementos independientes de los propios trabajos expuestos, resulta un ejercicio complejo. En general, los mejores trabajos escritos son presentados con mejor criterio y habilidad que aquellos de menor calidad. En la medida de lo posible, sin embargo, las calificaciones intentan reflejar entre otros aspectos la capacidad (y el esfuerzo) de (i) transmitir la información de forma clara, coherente y estructurada; (ii) separar lo relevante de lo accesorio; (iii) sintetizar la información; (iv) captar la atención de la audiencia; y (v) generar y gestionar el posterior debate.

Finalmente, también se incorpora a la evaluación un componente de desempeño individual basado en dos factores fundamentales. Por una parte, dado que todos los estudiantes han tenido la oportunidad (pero no la obligación) de participar en varias presentaciones, se tiene en cuenta el número de presentaciones en las que han 
participado. Por otro lado, también se valora la participación activa y constructiva de los estudiantes en las (i) discusiones y debates en el aula; (ii) charlas con emprendedores; y (iii) reuniones de supervisión y seguimiento con los profesores. Esto requiere un esfuerzo adicional de seguimiento por parte de los profesores, pues deben prestar atención al contenido de cada sesión, participar cuando así lo consideran necesario y registrar el grado y la calidad de las intervenciones de cada estudiante. ${ }^{25}$

\subsection{Resultados académicos}

Las calificaciones finales otorgadas a los estudiantes en esta asignatura han sido bastante elevadas y homogéneas, siendo las calificaciones mínima y máxima de 6,9 y 8,6, respectivamente, y la calificación media de 7,9 (en una escala de 0 a 10). En ese sentido, por encima de los registros numéricos, todos los profesores de la asignatura hemos percibido que la calidad de los trabajos, la calidad de las presentaciones y en definitiva el aprendizaje han sido muy satisfactorios. Y, lo que es más importante, todos los profesores compartimos la idea de que el nivel de participación, compromiso, motivación y esfuerzo por parte de los estudiantes ha sido encomiable.

\section{Diseño de la investigación}

Como hemos adelantado previamente, el objetivo principal de este trabajo es describir y evaluar una experiencia de aprendizaje interactivo desarrollada en la asignatura Pequeñas Empresas e Iniciativa Empresarial, perteneciente al programa Máster en Iniciativa Empresarial, Estrategia y Economía de las Organizaciones, que se ofrece en la Facultad de Economía de la Universidad Erasmus de Rotterdam.

Con ese propósito se recoge la opinión de los 24 estudiantes de esta asignatura y los 5 profesores sobre la metodología de enseñanza-aprendizaje utilizada, el sistema de evaluación y la asignatura en sí misma. En este sentido, dado el objeto de estudio, los estudiantes podrían mostrar cierta tendencia a "suavizar" sus opiniones, si no se garantizaba su anonimato. Por ello, un análisis cuantitativo en base a un cuestionario anónimo pareció el formato más adecuado para recabar su opinión. Por el contrario, una estructura cualitativa, a través de una entrevista semi-estructurada, fue la opción seleccionada para plasmar la versión de los profesores sobre esta experiencia.

\subsection{Cuestionario}


Los 24 estudiantes de la asignatura cumplimentaron un cuestionario que fue estructurado en 7 bloques: (A) información general (3 ítems); (B) formación académica (3 ítems); (C) experiencia laboral (2 ítems); (D) metodologías de enseñanza-aprendizaje utilizadas previamente y sus preferencias en ese sentido (4 ítems); (E) opinión y satisfacción con la metodología de enseñanza-aprendizaje utilizada durante esta asignatura (19 ítems); (F) opinión y satisfacción con el sistema de evaluación utilizado durante esta asignatura (8 ítems); y (G) opinión y satisfacción general con la asignatura (5 ítems). ${ }^{26}$

Los bloques A, B, C y D estuvieron diseñados mayoritariamente sobre preguntas de elección dicotómica y múltiple. Además, los bloques $\mathrm{A}$ y $\mathrm{B}$ incluyeron algunas preguntas abiertas, cuando se trataron cuestiones como el año o el país de nacimiento, o el nombre de la institución en la que se cursó el Grado. Por el contrario, los bloques E, F y G estuvieron basados casi en su totalidad en preguntas de escala de opinión tipo Likert de 5 puntos (1: Totalmente de acuerdo; 2: De acuerdo; 3: Parcialmente de acuerdo; 4: En desacuerdo; y 5: Totalmente en desacuerdo).

Una vez recopilada la información, y teniendo en cuenta las excepciones ya descritas para los bloques $A$ y $B$, tal información fue transformada en variables discretas binarias (tomando los valores 0 o 1 ), o en variables discretas y ordenadas (pudiendo variar entre los valores 1 y 5). A continuación, hallamos los valores medios para las variables binarias (pudiendo interpretarse como el porcentaje de respuestas tipo 1) y los valores medios y la desviación estándar para las variables discretas y ordenadas. $^{27}$

\subsection{Entrevista semi-estructurada}

Los 5 profesores de la asignatura mantuvieron una reunión, que se estructuró bajo el formato de entrevista semi-estructurada. El "guion" de esta entrevista tenía el objetivo de contrastar las diferentes impresiones y opiniones de los profesores sobre las ventajas e inconvenientes de los siguientes aspectos de la asignatura: (i) metodología de enseñanza-aprendizaje, y (ii) sistema de evaluación. Durante esta reunión, se trataron cuestiones específicas tales como el cumplimiento de los objetivos planteados en la asignatura, la satisfacción con el esfuerzo desarrollado por los estudiantes, la satisfacción con su aprendizaje, los posibles errores en el diseño y/o desarrollo de la asignatura, el tiempo invertido en la preparación y supervisión de la asignatura, los 
problemas surgidos a la hora de incorporar el componente de desempeño individual de cada estudiante en la evaluación, la posibilidad de aplicar esta metodología en otras asignaturas de corte más teórico, en otras etapas del sistema educativo, o sobre grupos con menos experiencia en este sistema de enseñanza-aprendizaje, con menor motivación y/o nivel de conocimientos adquiridos, o sencillamente más numerosos.

\section{Resultados}

Esta sección se estructura en dos bloques diferenciados, que se corresponden con las subsecciones 4.1 y 4.2 . El primer bloque presenta y discute los resultados obtenidos a raíz del análisis de las respuestas de los estudiantes al cuestionario. El segundo resume las impresiones y reflexiones más relevantes de los profesores de la asignatura, como consecuencia de la reunión que mantuvieron (en formato de entrevista semi-estructurada).

\subsection{Resultados a raíz del cuestionario (estudiantes)}

Esta subsección se centra en el análisis de los resultados obtenidos a través de las respuestas de los estudiantes a los 4 últimos bloques del cuestionario (bloques D, $E, F$ y $G$ ), que son los que recogen las valoraciones y opiniones de los estudiantes en relación a su participación en esta asignatura. Estas respuestas no solo se analizan de forma global, sino que se proponen dos criterios adicionales de agrupación de estudiantes, con el objetivo de identificar posibles perfiles diferentes de opinión y valoración de los diferentes aspectos que esta asignatura engloba.

El primer criterio consiste en agrupar a los estudiantes según sus respuestas a la pregunta acerca de sus calificaciones durante sus estudios previos de Grado. Entendemos que los estudiantes con mejores niveles de formación podrían tener diferentes características y valores que les hagan percibir y valorar su aprendizaje de otro modo al que lo hace el resto de estudiantes. ${ }^{28}$ De este modo, los 15 estudiantes que declararon que sus calificaciones estaban en la media de sus compañeros (14) o por debajo (1) constituyen un primer grupo (MED), mientras que los 9 estudiantes que afirmaron haber obtenido calificaciones por encima de la media conforman el segundo grupo (SUP).

El segundo criterio se basa en la agrupación de los estudiantes según el lugar en que cursaron sus estudios de Grado. Consideramos en este caso que la decisión de 
incorporarse al Máster en Iniciativa Empresarial, Estrategia y Economía de las Organizaciones, cuando esta lleva aparejada la necesidad de cambiar de país, puede atender a criterios y expectativas diferentes que cuando el cruce de fronteras no es necesario. En consecuencia, también cabe esperar que estos estudiantes que han cambiado su país de residencia para poder cursar este Máster perciban y valoren su aprendizaje de un modo diferente al que lo hace el resto de estudiantes. Así, los 16 estudiantes (entre ellos 5 extranjeros) cuya formación previa fue adquirida en Holanda forman el primer grupo $(\mathrm{HOL})$, mientras que los 8 estudiantes restantes (todos ellos extranjeros) que cursaron el Grado en el extranjero constituyen el segundo (EXT).

\subsubsection{Metodología de enseñanza-aprendizaje y tipología de asignaturas: experiencia previa y preferencias - Bloque $D$ -}

La Tabla 3, que presentamos a continuación, muestra tanto las preguntas utilizadas en el cuestionario para este bloque $\mathrm{D}$ como los resultados obtenidos.

\begin{tabular}{|c|c|c|c|c|c|c|c|c|c|}
\hline \multirow{3}{*}{\multicolumn{5}{|c|}{$\begin{array}{l}\text { METODOLOGÍA DE ENSEÑANZA-APRENDIZAJE: } \\
\text { EXPERIENCIA PREVIA Y PREFERENCIAS } \\
\text { Consideremos dos metodologías de enseñanza-aprendizaje opuestas: } \\
\text { a. Una metodología basada mayoritariamente en trabajo individual, asistencia a } \\
\text { clase y evaluación por medio de un examen final. } \\
\text { b. Una metodología basada mayoritariamente en trabajo en equipo, participación } \\
\text { activa, realización de trabajos de manera autónoma y presentación de los } \\
\text { mismos en público. }\end{array}$}} & \multicolumn{5}{|c|}{$\begin{array}{l}\text { Valor medio (1-5) y } \\
\text { desviación estándar }\end{array}$} \\
\hline & & & & & \multirow[b]{2}{*}{ Total } & \multicolumn{2}{|c|}{$\begin{array}{l}\text { Resultados } \\
\text { académicos }\end{array}$} & \multicolumn{2}{|c|}{$\begin{array}{l}\text { Lugar en que se } \\
\text { cursó el Grado }\end{array}$} \\
\hline & & & & & & MED & SUP & HOL & EXT \\
\hline \multicolumn{5}{|c|}{$\begin{array}{l}\text { D1. Durante tus estudios de Grado, ¿qué metodología de enseñanza-aprendizaje } \\
\text { seguiste en la institución en la que estudiaste? }\end{array}$} & \multirow{2}{*}{$\begin{array}{c}2,25 \\
(1,19)\end{array}$} & \multirow{2}{*}{$\begin{array}{l}2,27 \\
(1,16)\end{array}$} & \multirow{2}{*}{$\begin{array}{l}2,22 \\
(1,3)\end{array}$} & \multirow{2}{*}{$\begin{array}{l}2,69 \\
(1,2)\end{array}$} & \multirow{2}{*}{$\begin{array}{l}1,38 \\
(0,52)\end{array}$} \\
\hline \begin{tabular}{c}
\multicolumn{1}{c}{$(1)$} \\
a: $90-100 \%$ \\
b: $0-10 \%$
\end{tabular} & $\begin{array}{c}(2) \\
\text { a: } 60-90 \% \\
\text { b: } 10-40 \%\end{array}$ & $\begin{array}{c}(3) \\
\text { a: } 40-60 \% \\
\text { b: } 40-60 \%\end{array}$ & $\begin{array}{c}(4) \\
\text { a: } 10-40 \% \\
\text { b: } 60-90 \%\end{array}$ & \begin{tabular}{|c|}
\multicolumn{1}{|c|}{$(5)$} \\
a: $0-10 \%$ \\
b: $90-100 \%$
\end{tabular} & & & & & \\
\hline \multicolumn{5}{|c|}{ D2. ¿Qué metodología de enseñanza-aprendizaje prefieres? } & \multirow[b]{2}{*}{$\begin{array}{c}3 \\
(1,02)\end{array}$} & \multirow[b]{2}{*}{$\begin{array}{c}3,13 \\
(1,06)\end{array}$} & \multirow[b]{2}{*}{$\begin{array}{c}2,78 \\
(0,97)\end{array}$} & \multirow[b]{2}{*}{$\begin{array}{l}2,88 \\
(1,02)\end{array}$} & \multirow[b]{2}{*}{$\begin{array}{l}3,25 \\
(1,04)\end{array}$} \\
\hline \begin{tabular}{c}
\multicolumn{1}{c}{$(1)$} \\
a: $90-100 \%$ \\
b: $0-10 \%$
\end{tabular} & $\begin{array}{c}(2) \\
\text { a: } 60-90 \% \\
\text { b: } 10-40 \%\end{array}$ & $\begin{array}{c}(3) \\
\text { a: } 40-60 \% \\
\text { b: } 40-60 \%\end{array}$ & $\begin{array}{c}(4) \\
\text { a: } 10-40 \% \\
\text { b: } 60-90 \%\end{array}$ & \begin{tabular}{|c|}
\multicolumn{1}{|c|}{$(5)$} \\
a: $0-10 \%$ \\
b: $90-100 \%$
\end{tabular} & & & & & \\
\hline \multirow{3}{*}{\multicolumn{5}{|c|}{$\begin{array}{l}\text { TIPOLOGÍA DE ASIGNATURAS: } \\
\text { EXPERIENCIA PREVIA Y PREFERENCIAS }\end{array}$}} & \multicolumn{5}{|c|}{ Valor medio $(0-1)$} \\
\hline & & & & & \multirow[b]{2}{*}{ Total } & \multicolumn{2}{|c|}{$\begin{array}{l}\text { Resultados } \\
\text { académicos }\end{array}$} & \multicolumn{2}{|c|}{$\begin{array}{l}\text { Lugar en que se } \\
\text { cursó el Grado }\end{array}$} \\
\hline & & & & & & MED & SUP & HOL & EXT \\
\hline \multicolumn{5}{|c|}{$\begin{array}{l}\text { D3. Durante tus estudios de Grado, ¿qué tipo de asignaturas cursaste? } \\
\text { 1. Asignaturas principalmente interdisciplinares: diferentes tópicos fueron } \\
\text { cubiertos de forma simultánea en la misma asignatura (como esta asignatura }\end{array}$} & 0,71 & 0,73 & 0,67 & 0,75 & 0,63 \\
\hline
\end{tabular}




\begin{tabular}{|c|c|c|c|c|c|c|}
\hline 0 & $\begin{array}{l}\text { por ejemplo, que abarca Economía, Econometría, Estrategia y Finanzas). } \\
\text { Asignaturas principalmente focalizadas, que se centran en una sola materia. }\end{array}$ & & & & & \\
\hline 1. & $\begin{array}{l}\text { ¿Qué tipo de asignaturas prefieres? } \\
\text { Asignaturas interdisciplinares: diferentes tópicos fueron cubiertos de forma } \\
\text { simultánea en la misma asignatura. } \\
\text { Asignaturas focalizadas, que se centran en una sola materia. }\end{array}$ & 0,7 & 0,79 & 0,56 & 0,75 & 0,57 \\
\hline
\end{tabular}

Tabla 3. Cuestionario correspondiente al bloque D y resumen de resultados. Fuente: Elaboración propia.

Como podemos ver, este bloque está compuesto por 4 ítems (D1-D4). El primer resultado interesante surge de comparar la metodología de enseñanza-aprendizaje utilizada por los estudiantes durante sus estudios de Grado y sus preferencias en este sentido. Así, parece desprenderse que los estudiantes han seguido metodologías con una mayor presencia relativa del trabajo individual, la clase magistral y la evaluación por medio de un examen final (valor medio en el ítem D1 para el total de estudiantes igual a 2,25). Sin embargo, la elevada dispersión en las respuestas de los estudiantes (dispersión en el ítem D1 para el total de estudiantes igual a 1,19) exige de cierta cautela a la hora de valorar este dato. Esta dispersión parece explicarse (al menos de manera parcial) cuando tenemos en cuenta el lugar en que se cursaron los estudios de Grado. Así, aquellos estudiantes cuya formación previa fue adquirida en Holanda (HOL) parecen haber seguido sistemas mixtos, con mayor o menor peso del aprendizaje autónomo y la clase magistral (valor medio en el ítem D1 igual a 2,69). Por el contrario, en los estudiantes que cursaron el Grado en el extranjero (EXT) han primado los sistemas individualistas basados en un examen final (valor medio en el ítem D1 igual a 1,38).

En este sentido, identificar unas diferencias tan marcadas en los sistemas educativos previos entre las categorías HOL y EXT servirá de base para explicar algunas de las diferencias que se identifican en las respuestas al cuestionario al comparar ambos grupos de estudiantes. En otras palabras, este criterio de clasificación de estudiantes permite no solo identificar los efectos de las expectativas existentes cuando se toma la decisión de cambiar de país para realizar unos estudios, sino también enfrentar el efecto que causan los diferentes sistemas educativos previos.

Con respecto a sus preferencias acerca del sistema educativo a seguir, los estudiantes se decantan por sistemas mixtos, basados tanto en el trabajo individual como en equipo, tanto en la asistencia a clase como en la participación activa, y tanto 
en la realización de un examen final como en la realización de trabajos de manera autónoma y su posterior presentación en público (valor medio en el ítem D2 igual a 3, con una dispersión moderada igual a 1,02). Sin embargo, los criterios de clasificación de estudiantes MED-SUP y HOL-EXT arrojan diferencias interesantes. Así, son los estudiantes de tipo MED (frente a los de tipo SUP; valores medios en el ítem D2 iguales a 3,13 y 2,78 respectivamente) y los de tipo EXT (frente a los de tipo HOL; valores medios en el ítem D2 iguales a 3,25 y 2,88 respectivamente) los que muestran mayor afinidad con los sistemas de aprendizaje interactivos. Estas divergencias, aunque moderadas, podrían estar revelando las ventajas que aprecian los estudiantes de tipo MED a la hora de preparar una asignatura, adquirir conocimientos y ser evaluados en base al trabajo en equipo. Así, estos equipos donde participan otros estudiantes (en muchos casos) más dotados podría beneficiarles tanto en términos de aprendizaje como en su calificación final. Del mismo modo, los estudiantes de tipo EXT, habiendo seguido sistemas más individualistas, y teniendo por tanto un mejor conocimiento de las características de ambos sistemas de aprendizaje, parecen apreciar en mayor medida las características de los sistemas más cooperativos.

En lo relativo al contenido de las asignaturas, el $70 \%$ de los estudiantes parece preferir y estar habituado a temarios de tipo interdisciplinar en los que se cubren diferentes tópicos de forma simultánea más que temarios muy focalizados que se centran en una sola materia (ítems D3 y D4). De los criterios de clasificación de estudiantes MED-SUP y HOL-EXT no parecen desprenderse diferencias relevantes en este sentido.

\subsubsection{Evaluando la metodología de enseñanza-aprendizaje de la asignatura - Bloque E -}

A continuación, mostramos la Tabla 4, que presenta las preguntas y los resultados obtenidos para este bloque $\mathrm{E}$ destinado a cubrir la opinión de los estudiantes sobre la metodología de enseñanza-aprendizaje seguida durante esta asignatura.

\begin{tabular}{|c|c|c|c|c|c|}
\hline \multirow{3}{*}{ AFIRMACIONES A VALIDAR O RECHAZAR POR LOS ESTUDIANTES } & \multicolumn{5}{|c|}{$\begin{array}{l}\text { Valor medio (1-5) y } \\
\text { desviación estándar }\end{array}$} \\
\hline & & \multicolumn{2}{|c|}{$\begin{array}{l}\text { Resultados } \\
\text { académicos }\end{array}$} & \multicolumn{2}{|c|}{$\begin{array}{l}\text { Lugar en que } \\
\text { se cursó el } \\
\text { Grado }\end{array}$} \\
\hline & Total & MED & SUP & HOL & EXT \\
\hline
\end{tabular}


Millán, J.M., Millán, A. y Román, C.: Estudio de una experiencia internacional de aprendizaje interactivo en la asignatura

\begin{tabular}{|c|c|c|c|c|c|}
\hline \multicolumn{6}{|l|}{ Idioma (Inglés) } \\
\hline $\begin{array}{l}\text { E1. Tener que utilizar una lengua diferente (Inglés) a mi lengua materna ha } \\
\text { sido incómodo. } \\
\text {-No respondas a esta pregunta si tu lengua materna es el inglés- }\end{array}$ & $\begin{array}{l}4,04 \\
(1,3)\end{array}$ & $\begin{array}{c}3,64 \\
(1,45)\end{array}$ & $\begin{array}{c}4,67 \\
(0,71)\end{array}$ & $\begin{array}{l}4,27 \\
(1,1)\end{array}$ & $\begin{array}{l}3,63 \\
(1,6)\end{array}$ \\
\hline \multicolumn{6}{|l|}{ Sesiones introductorias } \\
\hline $\begin{array}{l}\text { E2. Las sesiones introductorias fueron suficientemente informativas para } \\
\text { poder empezar a trabajar en equipo de forma autónoma. }\end{array}$ & $\begin{array}{c}2,7 \\
(0,97)\end{array}$ & $\begin{array}{l}2,71 \\
(0,99)\end{array}$ & $\begin{array}{r}2,67 \\
(1)\end{array}$ & $\begin{array}{c}2,94 \\
(1)\end{array}$ & $\begin{array}{l}2,14 \\
(0,69)\end{array}$ \\
\hline \multicolumn{6}{|l|}{ Trabajo en equipo } \\
\hline $\begin{array}{l}\text { E3. Trabajar en equipo con mis compañeros ha sido positivo para mi } \\
\text { aprendizaje. }\end{array}$ & $\begin{array}{c}2,29 \\
(1)\end{array}$ & $\begin{array}{l}2,13 \\
(0,52)\end{array}$ & $\begin{array}{c}2,56 \\
(1,51)\end{array}$ & $\begin{array}{c}2,38 \\
(0,89)\end{array}$ & $\begin{array}{c}2,13 \\
(1,25)\end{array}$ \\
\hline $\begin{array}{l}\text { E4. Trabajando en equipo lo pasamos bien y nuestra relación siempre fue } \\
\text { excelente. }\end{array}$ & $\begin{array}{l}2,22 \\
(1,09)\end{array}$ & $\begin{array}{c}2 \\
(0,68)\end{array}$ & $\begin{array}{c}2,56 \\
(1,51)\end{array}$ & $\begin{array}{l}2,33 \\
(0,9)\end{array}$ & $\begin{array}{c}2 \\
(1,41)\end{array}$ \\
\hline $\begin{array}{l}\text { E5. Las diferencias existentes en el tipo de formación académica entre los } \\
\text { miembros de los diferentes equipos en los que he participado han sido } \\
\text { positivas para nuestro desempeño. }\end{array}$ & $\begin{array}{l}2,67 \\
(1,24)\end{array}$ & $\begin{array}{l}2,47 \\
(1,06)\end{array}$ & $\begin{array}{c}3 \\
(1,5)\end{array}$ & $\begin{array}{c}2,88 \\
(1,15)\end{array}$ & $\begin{array}{l}2,25 \\
(1,39)\end{array}$ \\
\hline $\begin{array}{l}\text { E6. La existencia de diferentes nacionalidades entre los miembros de los } \\
\text { diferentes equipos en los que he participado ha sido positiva para nuestro } \\
\text { desempeño. }\end{array}$ & $\begin{array}{l}2,67 \\
(1,27)\end{array}$ & $\begin{array}{l}2,73 \\
(1,22)\end{array}$ & $\begin{array}{c}2,56 \\
(1,42)\end{array}$ & $\begin{array}{l}3,06 \\
(1,24)\end{array}$ & $\begin{array}{l}1,88 \\
(0,99)\end{array}$ \\
\hline \multicolumn{6}{|l|}{ Profesores } \\
\hline $\begin{array}{l}\text { E7. La presencia de múltiples profesores en la asignatura ha sido positiva } \\
\text { para mi aprendizaje. }\end{array}$ & $\begin{array}{c}2,5 \\
(1,02)\end{array}$ & $\begin{array}{l}2,33 \\
(0,98)\end{array}$ & $\begin{array}{c}2,78 \\
(1,09)\end{array}$ & $\begin{array}{c}2,75 \\
(1,06)\end{array}$ & $\begin{array}{c}2 \\
(0,76)\end{array}$ \\
\hline $\begin{array}{l}\text { E8. Recibimos una orientación útil durante nuestras reuniones de } \\
\text { tutorización y seguimiento con los profesores. }\end{array}$ & $\begin{array}{l}2,09 \\
(0,95)\end{array}$ & $\begin{array}{l}2,07 \\
(0,92)\end{array}$ & $\begin{array}{l}2,11 \\
(1,05)\end{array}$ & $\begin{array}{l}2,19 \\
(1,05)\end{array}$ & $\begin{array}{c}1,86 \\
(0,69)\end{array}$ \\
\hline \multicolumn{6}{|l|}{ Presentaciones } \\
\hline $\begin{array}{l}\text { E9. Preparar presentaciones nos ayudó a distinguir las cuestiones } \\
\text { relevantes de las accesorias en los trabajos que realizamos. }\end{array}$ & $\begin{array}{c}2,17 \\
(0,76)\end{array}$ & $\begin{array}{c}2,33 \\
(0,82)\end{array}$ & $\begin{array}{l}1,89 \\
(0,6)\end{array}$ & $\begin{array}{l}2,31 \\
(0,7)\end{array}$ & $\begin{array}{c}1,88 \\
(0,83)\end{array}$ \\
\hline $\begin{array}{l}\text { E10. Recibimos comentarios útiles de profesores y compañeros durante } \\
\text { las presentaciones de los trabajos que realizamos. }\end{array}$ & $\begin{array}{l}2,71 \\
(0,91)\end{array}$ & $\begin{array}{l}2,67 \\
(0,98)\end{array}$ & $\begin{array}{c}2,78 \\
(0,83)\end{array}$ & $\begin{array}{c}3 \\
(0,89)\end{array}$ & $\begin{array}{l}2,13 \\
(0,64)\end{array}$ \\
\hline \multicolumn{6}{|l|}{ Trabajo de investigación en Iniciativa Empresarial } \\
\hline $\begin{array}{l}\text { E11. Haciendo un "Trabajo de Investigación en Iniciativa Empresarial" } \\
\text { aprendí mucho del tema específico sobre el que versó este trabajo. }\end{array}$ & $\begin{array}{l}2,21 \\
(1,1)\end{array}$ & $\begin{array}{l}2,33 \\
(1,18)\end{array}$ & $\begin{array}{l}2 \\
(1)\end{array}$ & $\begin{array}{c}2,38 \\
(1,09)\end{array}$ & $\begin{array}{c}1,88 \\
(1,13)\end{array}$ \\
\hline $\begin{array}{l}\text { E12. Tener que buscar e identificar nuestra propia bibliografía fue útil e } \\
\text { interesante en términos de aprendizaje. }\end{array}$ & $\begin{array}{c}2,42 \\
(1,02)\end{array}$ & $\begin{array}{l}2,47 \\
(0,92)\end{array}$ & $\begin{array}{c}2,33 \\
(1,22)\end{array}$ & $\begin{array}{c}2,56 \\
(1,03)\end{array}$ & $\begin{array}{l}2,13 \\
(0,99)\end{array}$ \\
\hline \multicolumn{6}{|l|}{ Charlas con emprendedores } \\
\hline $\begin{array}{l}\text { E13. Las charlas con los emprendedores fueron interesantes y útiles en } \\
\text { términos de aprendizaje. }\end{array}$ & $\begin{array}{c}2,54 \\
(1,32)\end{array}$ & $\begin{array}{c}2,2 \\
(1,01)\end{array}$ & $\begin{array}{l}3,11 \\
(1,62)\end{array}$ & $\begin{array}{c}3 \\
(1,32)\end{array}$ & $\begin{array}{c}1,63 \\
(0,74)\end{array}$ \\
\hline E14. Las charlas con los emprendedores fueron divertidas y emocionantes. & $\begin{array}{l}2,33 \\
(1,2)\end{array}$ & $\begin{array}{l}2,17 \\
(0,94)\end{array}$ & $\begin{array}{c}2,56 \\
(1,51)\end{array}$ & $\begin{array}{c}2,67 \\
(1,23)\end{array}$ & $\begin{array}{c}1,5 \\
(0,55)\end{array}$ \\
\hline \multicolumn{6}{|l|}{ Visita al centro de investigación EIM Business and Policy Research } \\
\hline $\begin{array}{l}\text { E15. La visita al centro de investigación fuer interesante y útil en términos } \\
\text { de aprendizaje. }\end{array}$ & $\begin{array}{l}3,17 \\
(1,31)\end{array}$ & $\begin{array}{l}2,93 \\
(1,39)\end{array}$ & $\begin{array}{l}3,56 \\
(1,13)\end{array}$ & $\begin{array}{l}3,63 \\
(1,2)\end{array}$ & $\begin{array}{c}2,25 \\
(1,04)\end{array}$ \\
\hline E16. La visita al centro de investigación fue divertida y $€$ & $\begin{array}{l}2,88 \\
(1,3)\end{array}$ & $\begin{array}{l}2,53 \\
(1,19)\end{array}$ & $\begin{array}{c}3,44 \\
(1,33)\end{array}$ & $\begin{array}{c}3,19 \\
(1,28)\end{array}$ & $\begin{array}{c}2,25 \\
(1,16)\end{array}$ \\
\hline
\end{tabular}




\begin{tabular}{|c|c|c|c|c|c|}
\hline \multicolumn{6}{|l|}{ Trabajo sobre Estrategia Empresarial } \\
\hline $\begin{array}{l}\text { E17. El "Trabajo sobre Estrategia Empresarial" fue interesante y útil en } \\
\text { términos de aprendizaje. }\end{array}$ & $\begin{array}{c}2,08 \\
(0,97)\end{array}$ & $\begin{array}{c}2,13 \\
(0,92)\end{array}$ & $\begin{array}{c}2 \\
(1,12)\end{array}$ & $\begin{array}{l}2,38 \\
(0,89)\end{array}$ & $\begin{array}{c}1,5 \\
(0,93)\end{array}$ \\
\hline \multicolumn{6}{|l|}{ Trabajo sobre Valoración Empresarial } \\
\hline $\begin{array}{l}\text { E18. El "Trabajo sobre Valoración Empresarial" fue interesante y útil en } \\
\text { términos de aprendizaje. }\end{array}$ & $\begin{array}{l}1,88 \\
(0,8)\end{array}$ & $\begin{array}{c}1,93 \\
(0,59)\end{array}$ & $\begin{array}{c}1,78 \\
(1,09)\end{array}$ & $\begin{array}{c}2,13 \\
(0,81)\end{array}$ & $\begin{array}{c}1,38 \\
(0,52)\end{array}$ \\
\hline \multicolumn{6}{|l|}{ Satisfacción general con la metodología de enseñanza-aprendizaje } \\
\hline $\begin{array}{l}\text { E19. En general, estoy satisfecho con la metodología de enseñanza- } \\
\text { aprendizaje utilizada en esta asignatura. }\end{array}$ & $\begin{array}{c}2,04 \\
(0,69)\end{array}$ & $\begin{array}{c}2 \\
(0,76)\end{array}$ & $\begin{array}{l}2,11 \\
(0,6)\end{array}$ & $\begin{array}{c}2,31 \\
(0,48)\end{array}$ & $\begin{array}{c}1,5 \\
(0,76)\end{array}$ \\
\hline
\end{tabular}

Tabla 4. Cuestionario correspondiente al bloque E y resumen de resultados. Fuente: Elaboración propia.

Este es el bloque más extenso del cuestionario, compuesto por 19 ítems (E1E19). Como vemos, los estudiantes no piensan en absoluto que haber utilizado una lengua diferente (en este caso la lengua inglesa) a su lengua materna haya sido algo incómodo (valor medio en el ítem E1 igual a 4,04). Sin embargo, los criterios de clasificación de estudiantes MED-SUP y HOL-EXT nos muestran cómo los estudiantes de tipo SUP (frente a los de tipo MED; valores medios en el ítem E1 iguales a 4,67 y 3,64 respectivamente) y los de tipo HOL (frente a los de tipo EXT; valores medios en el ítem E1 iguales a 4,27 y 3,63 respectivamente) muestran menores reticencias al uso de esta lengua, probablemente por tener un mejor conocimiento de la misma. En cualquier caso, estos resultados son coherentes con los beneficios de la internacionalización de la educación superior que sugieren Hénard et al. (2012).

En cuanto a las sesiones introductorias al trabajo a realizar, estas no son percibidas ni como suficientes ni insuficientes para empezar a trabajar de manera autónoma (valor medio en el ítem E2 igual a 2,7). Sin embargo, los estudiantes tipo HOL, más habituados a este sistema de trabajo, y por tanto más exigentes tal vez que los de tipo EXT, parecen estar menos satisfechos con estas sesiones introductorias (valores medios en el ítem E2 iguales a 2,94 y 2,14). En este caso, al contrario de lo que ocurre con otros aspectos de esta modalidad de "clase invertida" como veremos más adelante, parece que la implementación de estas sesiones introductorias demanda cierta reflexión.

El trabajo en equipo, recurso habitual de estos entornos de aprendizaje constructivistas (Jonassen, 1991; Kafai \& Resnick, 1996), sí parece ser percibido por los estudiantes como muy beneficioso, tanto para el aprendizaje (valor medio en el ítem 
E3 igual a 2,29) como desde el punto de vista de lo ameno del sistema (valor medio en el ítem E4 igual a 2,22). No obstante, los estudiantes de tipo SUP (frente a los de tipo MED) parecen ser los que perciben ventajas moderadamente menores de este trabajo en equipo (valores medios iguales a 2,56 en ambos ítem E3 y E4 para los de tipo SUP), lo cual es coherente con lo obtenido previamente en el ítem D2.

Los estudiantes no parecen percibir de manera unánime el efecto que sobre su aprendizaje genera la heterogeneidad en la formación académica de los miembros del grupo (valor medio en el ítem E5 igual a 2,67 con dispersión elevada igual a 1,24). Nuevamente son los estudiantes de tipo SUP (frente a los de tipo MED) los que tienen una visión menos positiva sobre esta heterogeneidad (valores medios en el ítem E5 iguales a 3 y 2,47 respectivamente). Estas diferencias que emergen siguen la línea de lo obtenido en los ítems D2, E3 y E4. Por otra parte, en coherencia con lo obtenido en el ítem E2, los estudiantes de tipo EXT (frente a los de tipo HOL) sí parecen apreciar ventajas de esta heterogeneidad (valores medios en el ítem E5 iguales a 2,25 y 2,88 respectivamente).

En el caso del factor nacionalidad y su incidencia sobre el aprendizaje (ítem E6), los resultados no parecen ser concluyentes si tan solo atendemos al dato global (valor medio en el ítem E6 igual a 2,67 con dispersión elevada igual a 1,27). En este caso, la elevada dispersión parece provenir del criterio de clasificación HOL-EXT. Así, los estudiantes de tipo EXT (frente a los de tipo HOL) parecen ser un grupo que sí percibe la existencia de múltiples nacionalidades en los grupos de trabajo como un atributo positivo para el desempeño académico del propio grupo (valores medios en el ítem E6 iguales a 1,88 y 3,06 respectivamente). Cabe destacar que estamos enfrentando los resultados de dos grupos muy dispares. De un lado, un grupo que ha cursado sus estudios de Grado en Holanda (HOL), en atmósferas habitualmente muy internacionales, y que por tanto si bien conocen sus ventajas, no perciben este atributo como una novedad. Del otro, un grupo cuya inmersión internacional es probablemente menor (EXT), pero que ha elegido expresamente este formato de dimensión internacional para culminar sus estudios universitarios, y que por tanto valora especialmente las bondades de la internacionalización de la educación superior que sugieren Hénard et al. (2012).

La presencia de múltiples profesores ha sido percibida de forma moderadamente positiva (valor medio en el ítem E7 igual a 2,5), siendo el grupo de estudiantes tipo EXT (frente a los de tipo $\mathrm{HOL}$ ) los que parecen valorar en mayor medida esta característica 
de la asignatura (valores medios en el ítem E7 iguales a 2 y 2,75 respectivamente). En cuanto a la interacción con los profesores durante las reuniones de tutorización y seguimiento, y en coherencia con los efectos positivos de estas actividades que la literatura identifica (Cohen et al., 1982), esta ha sido valorada como muy útil (valor medio en el ítem E8 igual a 2,09), no apreciándose diferencias relevantes a través de los criterios de clasificación de estudiantes MED-SUP y HOL-EXT.

En consonancia con lo apuntado por Torres-Pérez (2012), el proceso de preparación de las presentaciones en público ha favorecido en los estudiantes el desarrollo de competencias importantes como la distinción entre las cuestiones más relevantes y trascendentes de sus propios trabajos de las más accesorias (valor medio en el ítem E9 igual a 2,17). En esa misma línea, los estudiantes parecen percibir de forma moderadamente positiva los beneficios de los comentarios por parte de profesores y compañeros, recibidos durante sus presentaciones (valor medio en el ítem E10 igual a 2,5). Para ambos ítems, y tal y como ya ocurriera para los ítems E2 y E5, vuelve a ser el grupo de estudiantes tipo EXT (frente a los de tipo HOL) el que percibe de forma más positiva la preparación de presentaciones, y el feedback recibido durante las mismas (valores medios en los ítem E9 y E10 para los estudiantes tipo EXT iguales a 1,88 y 2,13 respectivamente).

La realización de un "Trabajo de Investigación en Iniciativa Empresarial” parece constituir uno de los puntales del aprendizaje (valor medio en el ítem E11 igual a 2,21), así como la revisión de las distintas fuentes bibliográficas durante su realización (valor medio en el ítem E12 igual a 2,42). Además, los criterios de clasificación de estudiantes MED-SUP y HOL-EXT no parecen arrojar diferencias significativas. La robustez de estos resultados parece dar soporte a la conveniencia de incorporar en los procesos de enseñanza-aprendizaje aquellos recursos didácticos aparejados al desarrollo de esta actividad, esto es, el "aprendizaje basado en proyectos" (Nunes de Oliveira, 2011) y el denominado "escribir para aprender" (Emig, 1977; Román et al., 2016).

Las charlas con verdaderos emprendedores son objeto de una evaluación moderadamente positiva por parte de los estudiantes en términos de su contribución al aprendizaje (valor medio en el ítem E13 igual a 2,54). Sin embargo, encontramos bastante heterogeneidad en las respuestas de los estudiantes (dispersión en el ítem E13 igual a 1,32), siendo los grupos de estudiantes tipo SUP (frente a los de tipo MED) y HOL (frente a los de tipo EXT) los que encuentran estas charlas menos relevantes (valores medios en el ítem E13 para SUP y HOL iguales a 3,11 y 3, respectivamente). 
Por el contrario, lo ameno de estas charlas no parece estar en tela de juicio por parte de los estudiantes (valor medio en el ítem E14 igual a 2,33). Sin embargo, el grupo de estudiantes tipo EXT (frente a los de tipo HOL) parece haber disfrutado más con esta actividad (valores medios en el ítem E14 iguales a 1,5 y 2,67 respectivamente).

En cuanto a la visita al centro de investigación EIM Business and Policy Research, los estudiantes no parecen estar muy de acuerdo en relación a los aspectos positivos de la misma, ni en relación con el aprendizaje (valor medio en el ítem E15 igual a 3,17 con elevada dispersión igual a 1,31) ni con lo ameno de la visita (valor medio en el ítem E16 igual a 2,88 con elevada dispersión igual a 1,3). Nuevamente, son los estudiantes tipo SUP (frente a los de tipo MED) y tipo HOL (frente a los de tipo EXT) los que reflejan posturas más desfavorables en relación a esta actividad (valores medios en los ítem E15 y E16 iguales a 3,56 y 3,44 para estudiantes tipo SUP, e iguales a 3,63 y 3,19 para estudiantes tipo HOL). Reflexionar sobre el diseño de este tipo de visitas se revela por tanto como necesario, a la luz de estos resultados.

Los "Trabajos sobre Estrategia Empresarial” (valor medio en el ítem E17 igual a 2,08) y sobre "Valoración Empresarial" (valor medio en el ítem E18 igual a 1,88) también parecen ser muy apreciados por los estudiantes por su contribución al aprendizaje. En este sentido, destaca la percepción favorable de los estudiantes tipo EXT (frente a los de tipo HOL) sobre estos trabajos (valores medios en los ítems E17 y E18 iguales a 1,5 y 1,38 para estudiantes tipo EXT). En cualquier caso, estos resultados vuelven a respaldar las bondades de recursos didácticos como el "aprendizaje basado en proyectos" (Nunes de Oliveira, 2011) y el denominado "escribir para aprender" (Emig, 1977; Román et al., 2016).

Finalmente, y tras evaluar de manera independiente todos estos aspectos de la metodología de enseñanza-aprendizaje de tipo constructivista utilizada en esta asignatura, los estudiantes expresan una gran satisfacción con la misma considerada de forma global (valor medio en el ítem E19 igual a 2,04). Como era de esperar, tras los resultados obtenidos en la valoración de los aspectos individuales de la metodología, los estudiantes de tipo EXT (frente a los de tipo HOL) conforman el grupo con mayor nivel de satisfacción global (valor medio en el ítem E19 igual a 1,5). En todo caso, estos resultados dan soporte a los trabajos que sugieren la aparición de mejoras en los procesos de aprendizaje mediante el uso de sistemas basados en la "clase invertida" (Crouch \& Mazur, 2001; Fagen et al., 2002). 


\subsubsection{Evaluando el sistema de calificación de la asignatura - Bloque F -}

La Tabla 5 mostrada a continuación presenta el cuestionario y los resultados obtenidos para el bloque $\mathrm{F}$, que se centra en la evaluación que los estudiantes hacen sobre el sistema de calificación de la asignatura.

\begin{tabular}{|c|c|c|c|c|c|c|c|c|c|}
\hline \multirow{3}{*}{\multicolumn{5}{|c|}{$\begin{array}{l}\text { PREFERENCIAS EN EL SISTEMA DE EVALUACIÓN } \\
\text { Consideremos dos sistemas de evaluación opuestos: } \\
\text { a. Un sistema basado principalmente en tu desempeño individual en un examen } \\
\text { final. } \\
\text { b. Un sistema basado principalmente en la participación activa, la realización de } \\
\text { trabajos de manera autónoma y su posterior presentación en público. }\end{array}$}} & \multicolumn{5}{|c|}{$\begin{array}{l}\text { Valor medio (1-5) y } \\
\text { desviación estándar }\end{array}$} \\
\hline & & & & & \multirow[b]{2}{*}{ Total } & \multicolumn{2}{|c|}{$\begin{array}{l}\text { Resultados } \\
\text { académicos }\end{array}$} & \multicolumn{2}{|c|}{$\begin{array}{l}\text { Lugar en que se } \\
\text { cursó el Grado }\end{array}$} \\
\hline & & & & & & MED & SUP & HOL & EXT \\
\hline \multicolumn{5}{|c|}{ F1. ¿Qué sistema de evaluación prefieres? } & \multirow[b]{2}{*}{$\begin{array}{c}3,32 \\
(1,29)\end{array}$} & \multirow[b]{2}{*}{$\begin{array}{c}3,36 \\
(1,45)\end{array}$} & \multirow[b]{2}{*}{$\begin{array}{l}3,25 \\
(1,04)\end{array}$} & \multirow[b]{2}{*}{$\begin{array}{c}3,33 \\
(1,18)\end{array}$} & \multirow[b]{2}{*}{$\begin{array}{l}3,29 \\
(1,6)\end{array}$} \\
\hline \begin{tabular}{l}
\multicolumn{1}{c}{$(1)$} \\
a: $90-100 \%$ \\
b: $0-10 \%$
\end{tabular} & $\begin{array}{c}(2) \\
\text { a: } 60-90 \% \\
\text { b: } 10-40 \%\end{array}$ & $\begin{array}{c}(3) \\
\text { a: } 40-60 \% \\
\text { b: } 40-60 \%\end{array}$ & $\begin{array}{c}(4) \\
\text { a: } 10-40 \% \\
\text { b: } 60-90 \%\end{array}$ & $\begin{array}{c}\text { (5) } \\
\text { a: } 0-10 \% \\
\text { b: } 90-100 \%\end{array}$ & & & & & \\
\hline \multicolumn{10}{|c|}{ AFIRMACIONES A VALIDAR O RECHAZAR POR LOS ESTUDIANTES } \\
\hline \multicolumn{10}{|c|}{ Calificación final } \\
\hline \multicolumn{5}{|c|}{ F2. Mi calificación final infravalora mi dedicación y esfuerzo. } & $\begin{array}{c}2,96 \\
(1,23)\end{array}$ & $\begin{array}{c}3,33 \\
(1,11)\end{array}$ & $\begin{array}{c}2,33 \\
(1,22)\end{array}$ & $\begin{array}{c}3,13 \\
(1,09)\end{array}$ & $\begin{array}{c}2,63 \\
(1,51)\end{array}$ \\
\hline \multicolumn{5}{|c|}{ F3. Mi calificación final infravalora mi grado de aprendizaje. } & $\begin{array}{l}3,21 \\
(1,1)\end{array}$ & $\begin{array}{c}3,33 \\
(0,98)\end{array}$ & $\begin{array}{c}3 \\
(1,32)\end{array}$ & $\begin{array}{c}3,38 \\
(0,89)\end{array}$ & $\begin{array}{c}2,88 \\
(1,46)\end{array}$ \\
\hline \multicolumn{5}{|c|}{$\begin{array}{l}\text { F4. Mi dedicación y esfuerzo hubiera sido mayor con un sistema de evaluación } \\
\text { basado principalmente en un examen final. }\end{array}$} & $\begin{array}{c}3,52 \\
(0,95)\end{array}$ & $\begin{array}{c}3,43 \\
(1,02)\end{array}$ & $\begin{array}{c}3,67 \\
(0,87)\end{array}$ & $\begin{array}{l}3,53 \\
(0,74)\end{array}$ & $\begin{array}{c}3,5 \\
(1,31)\end{array}$ \\
\hline \multicolumn{5}{|c|}{$\begin{array}{l}\text { F5. Mi grado de aprendizaje hubiera sido mayor con un sistema de evaluación } \\
\text { basado principalmente en un examen final. }\end{array}$} & $\begin{array}{c}3,67 \\
(1,01)\end{array}$ & $\begin{array}{c}3,6 \\
(1,06)\end{array}$ & $\begin{array}{c}3,78 \\
(0,97)\end{array}$ & $\begin{array}{c}3,63 \\
(0,89)\end{array}$ & $\begin{array}{c}3,75 \\
(1,28)\end{array}$ \\
\hline \multicolumn{5}{|c|}{$\begin{array}{l}\text { F6. Mi calificación final hubiera sido mayor con un sistema de evaluación basado } \\
\text { principalmente en un examen final. }\end{array}$} & $\begin{array}{l}3,52 \\
(1,1)\end{array}$ & $\begin{array}{c}3,63 \\
(1,04)\end{array}$ & $\begin{array}{c}3,33 \\
(1,22)\end{array}$ & $\begin{array}{c}3,66 \\
(0,87)\end{array}$ & $\begin{array}{c}3,25 \\
(1,49)\end{array}$ \\
\hline \multicolumn{10}{|c|}{ Satisfacción general con el sistema de evaluación } \\
\hline \multicolumn{5}{|c|}{ F7. En general, estoy satisfecho con el sistema de evaluación de esta asignatura. } & $\begin{array}{c}2,54 \\
(1,25)\end{array}$ & $\begin{array}{c}2,47 \\
(1,06)\end{array}$ & $\begin{array}{c}2,67 \\
(1,58)\end{array}$ & $\begin{array}{c}2,75 \\
(1,24)\end{array}$ & $\begin{array}{c}2,13 \\
(1,25)\end{array}$ \\
\hline \multicolumn{10}{|c|}{ Satisfacción general con la calificación final } \\
\hline \multicolumn{5}{|c|}{ F8. En general, estoy satisfecho con mi calificación final en esta asignatura. } & $\begin{array}{c}2,29 \\
(1,04)\end{array}$ & $\begin{array}{c}2,27 \\
(0,96)\end{array}$ & $\begin{array}{c}2,33 \\
(1,22)\end{array}$ & $\begin{array}{c}2,38 \\
(0,96)\end{array}$ & $\begin{array}{c}2,13 \\
(1,25)\end{array}$ \\
\hline
\end{tabular}

Tabla 5. Cuestionario correspondiente al bloque $\mathrm{F}$ y resumen de resultados. Fuente: Elaboración propia.

Este bloque está compuesto por 8 ítems (F1-F8). A través de las respuestas de los estudiantes al primer ítem (F1), vemos cómo éstos no parecen compartir sus 
preferencias por sistemas de evaluación concretos. Así, no parece imponerse ni el sistema tradicional basado principalmente en el desempeño individual en un examen final, ni tampoco el sistema basado principalmente en la participación activa, la realización de trabajos de manera autónoma y su posterior presentación en público. Estaríamos tentados a concluir que los estudiantes prefieren sistemas mixtos (valor medio en el ítem F1 igual a 3,32) si no fuera por la alta dispersión que observamos en esta variable (dispersión en el ítem F1 igual a 1,29), lo que nos lleva a interpretar los resultados en términos de falta de homogeneidad en las preferencias. Sin embargo, esta elevada dispersión no parece venir explicada a través de los criterios de clasificación de estudiantes MED-SUP y HOL-EXT, cuyas respuestas en relación a este ítem no arrojan diferencias significativas.

Podemos expresarnos en los mismos términos cuando consultamos a los estudiantes sobre su grado de afinidad con afirmaciones sobre si la calificación final de la asignatura infravalora tanto su esfuerzo como su grado de aprendizaje (ítems F2 y F3). Así, los estudiantes no parecen ni aceptar ni rechazar estas afirmaciones (valores medios en los ítems F2 y F3 iguales a 2,96 y 3,21, respectivamente) y estas variables también presentan dispersiones relativamente elevadas (dispersiones en los ítems F2 y F3 iguales a 1,23 y 1,1 respectivamente). En el caso de la posible infravaloración de la dedicación y el esfuerzo en la calificación final (ítem F2), sí podemos identificar una fuente de heterogeneidad a través del criterio de clasificación MED-SUP. Así, en la línea de los resultados presentes en los ítems D2, E3, E4 y E5, los estudiantes tipo SUP (frente a los de tipo MED) se revelan como claramente más de acuerdo con esta afirmación (valores medios en el ítem F2 iguales a 2,33 y 3,33 respectivamente).

Sin embargo, los estudiantes ofrecen respuestas más concentradas en torno a la media cuando afirman que no hubieran trabajado más (valor medio en el ítem F4 igual a 3,52), ni hubieran aprendido más (valor medio en el ítem F5 igual a 3,67) ni hubieran obtenido una mejor calificación (valor medio en el ítem F6 igual a 3,52) con un sistema de evaluación basado en el desempeño individual en un examen final, en comparación con el sistema de evaluación implementado en la asignatura (basado en participación activa, realización de trabajos de manera autónoma y presentación de los mismos en público). Además, estos resultados son robustos a la utilización de los criterios de clasificación de estudiantes MED-SUP y HOL-EXT (cuyas respuestas en relación a estos ítems siempre superan el valor 3 para todos los subgrupos). Estos resultados son consistentes con la literatura que defiende los beneficios de una evaluación continua 
entendida esta como un proceso planificado que permita la toma de conciencia por parte del alumno de su propio aprendizaje (Nicol \& Macfarlane-Dick, 2006; Weaver, 2006; Boud, 2007).

Finalmente, cuando los estudiantes son preguntados de forma general sobre su satisfacción con el sistema de evaluación (ítem F7), los estudiantes parecen estar tan solo relativamente satisfechos con el sistema de evaluación empleado (valor medio en el ítem F7 igual a 2,54). En este caso, una de las fuentes de la elevada dispersión registrada en las respuestas a este ítem (dispersión en el ítem F7 igual a 1,25) parece provenir de los estudiantes tipo EXT (frente a los de tipo HOL), que parecen mostrar mayores niveles de satisfacción (valores medios en el ítem F7 iguales a 2,13 y 2,75 respectivamente).

Menos dudas emergen sin embargo de los resultados obtenidos sobre la satisfacción de los estudiantes con la calificación final obtenida en la asignatura, con la que los estudiantes parecen estar satisfechos, ya sea considerando el grupo de estudiantes de manera global (valor medio en el ítem F8 igual a 2,29) como haciendo uso de los criterios de clasificación MED-SUP y HOL-EXT. ${ }^{29}$ Nuevamente se observa como nuestros resultados son coherentes con los trabajos que apuntan mejoras en el desempeño académico derivadas del uso de esta metodología de "clase invertida" (Crouch \& Mazur, 2001; Fagen et al., 2002).

\subsubsection{Satisfacción general con la asignatura - Bloque G -}

Finalmente, la Tabla 6 que aparece a continuación muestra el cuestionario y los resultados obtenidos para el bloque $\mathrm{G}$, dedicado a la evaluación de la asignatura de un modo global.

\begin{tabular}{|c|c|c|c|c|c|}
\hline \multirow{3}{*}{ AFIRMACIONES A VALIDAR O RECHAZAR POR LOS ESTUDIANTES } & \multicolumn{5}{|c|}{$\begin{array}{l}\text { Valor medio (1-5) y } \\
\text { desviación estándar }\end{array}$} \\
\hline & \multirow[b]{2}{*}{ Total } & \multicolumn{2}{|c|}{$\begin{array}{l}\text { Resultados } \\
\text { académicos }\end{array}$} & \multicolumn{2}{|c|}{$\begin{array}{l}\text { Lugar en que se } \\
\text { cursó el Grado }\end{array}$} \\
\hline & & MED & SuP & HOL & EXT \\
\hline \multicolumn{6}{|l|}{ Expectativas } \\
\hline $\begin{array}{l}\text { G1. Mis expectativas previas en términos de la calidad y utilidad de esta asignatura } \\
\text { eran elevadas. }\end{array}$ & $\begin{array}{l}2,29 \\
(0,91)\end{array}$ & $\begin{array}{l}2,07 \\
(0,7)\end{array}$ & $\begin{array}{l}2,67 \\
(1,12)\end{array}$ & $\begin{array}{l}2,56 \\
(0,89)\end{array}$ & $\begin{array}{l}1,75 \\
(0,71)\end{array}$ \\
\hline \multicolumn{6}{|l|}{ Calidad / Utilidad de la asignatura } \\
\hline G2. Cursar esta asignatura ha mejorado mi formación académica. & 2,17 & 2,07 & 2,33 & 2,44 & 1,63 \\
\hline
\end{tabular}




\begin{tabular}{|l|c|c|c|c|c|}
\hline & $(0,92)$ & $(0,96)$ & $(0,87)$ & $(0,89)$ & $(0,74)$ \\
\hline $\begin{array}{l}\text { G3. Cursar esta asignatura me ha aportado algunas de las habilidades necesarias } \\
\text { para el tipo de trabajo que estoy considerando para mi futuro profesional. }\end{array}$ & $\begin{array}{c}2,79 \\
(0,98)\end{array}$ & $\begin{array}{c}2,47 \\
(0,92)\end{array}$ & $\begin{array}{c}3,33 \\
(0,87)\end{array}$ & $\begin{array}{c}3,13 \\
(0,81)\end{array}$ & $\begin{array}{c}2,13 \\
(0,99)\end{array}$ \\
\hline G4. La calidad y utilidad de esta asignatura ha superado mis expectativas previas. & 2,88 & 2,67 & 3,22 & 3,19 & 2,25 \\
$(1,03)$ & $(1,05)$ & $(0,97)$ & $(0,83)$ & $(1,16)$ \\
\hline Satisfacción general con la asignatura & & 2,29 & 2,13 & 2,56 & 2,5 \\
\hline G5. En general, estoy satisfecho con la asignatura. & $(0,81)$ & $(0,74)$ & $(0,88)$ & $(0,73)$ & $(0,83)$ \\
\hline
\end{tabular}

Tabla 6. Cuestionario correspondiente al bloque $\mathrm{G}$ y resumen de resultados. Fuente: Elaboración propia.

El último bloque está compuesto por 5 ítems (G1-G5). En primer lugar, observamos cómo las expectativas generadas por la asignatura eran elevadas (valor medio en el ítem G1 igual a 2,29). Además, en opinión de los estudiantes, cursar la asignatura ha elevado su formación académica (valor medio en el ítem G2 igual a 2,17), lo que también es consistente con los trabajos que sugieren que los procesos de aprendizaje se ven favorecidos mediante el uso de estos sistemas constructivistas (Crouch \& Mazur, 2001; Fagen et al., 2002). En ambos casos, son los estudiantes de tipo EXT (frente a los de tipo HOL) los que sobremanera presentaban grandes expectativas (valores medios en el ítem $\mathrm{G} 1$ iguales a 1,75 y 2,56 respectivamente) y una mejor opinión en cuanto a la mejora de la formación académica alcanzada por medio de la asignatura (valores medios en el ítem G2 iguales a 1,63 y 2,44 respectivamente).

Sin embargo, un aspecto fundamental del nuevo EEES como es la contribución de la asignatura a las necesidades del mercado laboral (King \& Behnke, 2005) tan solo se percibe de una manera neutral (valor medio en el ítem G3 igual a 2,79), observándose nuevamente a los estudiantes de tipo EXT (frente a los de tipo HOL) como el grupo de opinión más favorable a las aportaciones de esta asignatura de cara a la incorporación al mercado laboral (valores medios en el ítem G3 iguales a 2,13 y 3,13 respectivamente).

Tampoco parece haber evidencias ni a favor ni en contra de que la asignatura haya superado las elevadas expectativas para los estudiantes, cuando estos son considerados de manera global (valor medio en el ítem G4 igual a 2,88). Sin embargo, los estudiantes de tipo EXT (frente a los de tipo HOL) vuelven a mostrar posiciones más 
favorables, en este caso, en relación al cumplimiento de las expectativas iniciales (valores medios en el ítem G4 iguales a 2,25 y 3,19 respectivamente).

Finalmente, a la hora de evaluar la asignatura de manera global, los estudiantes sí parecen estar bastante satisfechos con la misma (valor medio en el ítem G5 igual a $2,29)$, si bien, como ha venido siendo habitual a lo largo del cuestionario, los grupos de estudiantes tipo MED (frente a los de tipo SUP; valores medios iguales a 2,13 y 2,56, respectivamente) y EXT (frente a los de tipo HOL; valores medios iguales a 1,88 y 2,5, respectivamente) son los más satisfechos.

\subsection{Resultados a raíz de la entrevista semi-estructurada (profesores)}

Tal y como se ha apuntado previamente, esta entrevista recogió los puntos de vista de los docentes en relación a los siguientes aspectos de la asignatura: (i) metodología de enseñanza-aprendizaje, y (ii) sistema de evaluación. En este sentido, los profesores coinciden en señalar esta experiencia como un éxito global tanto metodológico como de resultados académicos. Además, los profesores valoran muy positivamente el esfuerzo, satisfacción y aprendizaje por parte de los estudiantes. Sin embargo, algunas reflexiones de carácter más particular, si bien no empañan la evaluación favorable de la experiencia por parte de los profesores, sí que merecen especial atención.

En primer lugar, en opinión de los profesores, el tener una sólida base de conocimientos adquirida durante sus estudios de Grado (lo que ocurría en una gran mayoría de los casos), junto con poseer cierta experiencia con sistemas de aprendizaje similares (lo que ocurría en todos los grupos de trabajo, al menos en algunos de sus componentes) parece haber favorecido los buenos resultados obtenidos. Sin embargo, la posibilidad de aplicar esta metodología en otras asignaturas de corte más teórico, o sobre grupos con menos experiencia en este sistema de enseñanza-aprendizaje, o sencillamente con menor motivación y/o formación parece un reto más complejo.

En segundo lugar, los profesores coinciden en señalar que la preparación y supervisión de esta asignatura supone un excesivo desgaste para el grupo de docentes, pese a contar con una reducida ratio alumnos-profesores (inferior a 5 alumnos por profesor). Por ello, mantener (o disminuir) esta ratio supone unos costes inasumibles para la mayoría de universidades, mientras que aumentarla generaría un 
mayor coste para el propio docente (ya de por sí elevadísimo) -y para la sociedad- en términos de reducción del tiempo disponible para sus propias labores de investigación.

Por último, los profesores han considerado la incorporación de un componente de desempeño individual de cada estudiante en la evaluación como el aspecto más complejo de este sistema de evaluación. Así, este sistema de evaluación tenía en cuenta la participación activa y constructiva de los estudiantes en las presentaciones, discusiones, charlas y debates en el aula, y en las reuniones de supervisión y seguimiento con los profesores. Sin embargo, el proceso de registro, además de laborioso e impreciso, está circunscrito al aula y a los despachos, y no permite capturar cómo ha sido el reparto de tareas entre los miembros del grupo para la realización de los trabajos en grupo, o cómo ha sido el trabajo individual desempeñado. Esta información asimétrica, sin duda, causa insatisfacción entre aquellos que consideran que su calificación final ha medido por defecto su esfuerzo y dedicación (lo cual parece haber ocurrido en los estudiantes de tipo SUP, tal y como muestran los resultados del ítem F2), y favorece el surgimiento del "problema del polizón".

\section{Conclusiones}

En este trabajo se describe y evalúa una experiencia internacional de aprendizaje interactivo desarrollada en una asignatura perteneciente a uno de los programas Máster que ofrece la Universidad Erasmus de Rotterdam. Los resultados obtenidos a través de esta experiencia educativa generan implicaciones relevantes de diversa índole.

En primer lugar, estos resultados son coherentes con los beneficios de la internacionalización de la educación superior que sugieren Hénard et al. (2012). En concreto, el ambiente internacional de esta experiencia y, sobre todo, su multilingüismo se revelan como elementos positivos en opinión de los estudiantes. En este sentido, ambos elementos vienen ya recogidos en la Estrategia para la Internacionalización de las Universidades Españolas 2015-2020 (Ministerio de Educación, Cultura y Deporte, 2014) dentro de las acciones específicas diseñadas en pro del objetivo de "Internacionalización en casa", esto es, para conseguir que los beneficios de la internacionalización alcancen también a la mayoría de estudiantes no móviles.

En segundo lugar, nuestros resultados también son congruentes con los trabajos de Crouch \& Mazur (2001) y Fagen et al. (2002), que sugieren que el uso de 
metodologías constructivistas diseñadas como una "clase invertida" genera mejoras notables en los procesos de aprendizaje y resultados académicos. En particular, los estudiantes perciben de manera muy positiva el uso de aquellos recursos didácticos que puedan fomentar el aprendizaje activo e interactivo, tales como el "aprendizaje basado en proyectos", "escribir para aprender", presentaciones orales o reuniones de tutorización y seguimiento. En este sentido, los estudiantes consideran que con esta metodología trabajan más, aprenden más y obtienen mejores calificaciones que con un sistema basado en trabajo individual y exámenes. Además, los estudiantes están satisfechos tanto con su calificación final como con la asignatura.

Sin embargo, incorporar al sistema educativo español algunas características de esta metodología de enseñanza-aprendizaje no es algo inmediato ni sencillo. De un lado, los docentes de esta experiencia coinciden en señalar como elementos favorecedores del éxito de esta experiencia la reducida ratio alumnos-profesores, la sólida preparación de los estudiantes adquirida durante sus estudios de Grado, el elevado nivel de motivación y la experiencia previa trabajando en equipo, lo que dificulta la posibilidad de implementar esta metodología en etapas anteriores del sistema educativo, en situaciones con estudiantes menos formados y/o motivados, o sencillamente en sistemas educativos dotados de menos recursos.

Además, el método de la "clase invertida" no está exento de detractores que lo consideran como una amenaza o sustituto de las clases presenciales (Luján-Mora, 2013). Paralelamente, desde una óptica institucional, e incluso desde el punto de vista del alumnado, podría considerarse que este método no es impartir docencia y que el profesor no está cumpliendo con sus obligaciones (Luján-Mora, 2013). No es menos cierto, sin embargo, que existen contraargumentos aparejados como que estos recursos deben entenderse en términos de complementariedad o recurso adicional en algunas asignaturas, y que permiten tanto aprovechar la clase presencial para realizar actividades en las que el papel del profesor es esencial como flexibilizar el ritmo de aprendizaje, de modo que cada alumno pueda ajustarlo a sus necesidades y ritmo.

En tercer lugar, el sistema de evaluación continua implementado, pese a haberse planificado e inspirado en el concepto de evaluación formativa, pese a ofrecer un buen nivel de desagregación, detalle y pautas, y pese a los esfuerzos de los docentes por incluir mecanismos de evaluación del trabajo individual, tan solo ha generado un nivel de satisfacción moderado por parte de los estudiantes, e incluso algunos estudiantes 
(en particular, los de tipo SUP) consideran que la calificación obtenida infravalora su dedicación y esfuerzo.

En este sentido, los docentes consideran que, si bien este sistema genera unos resultados más que satisfactorios en términos de esfuerzo, aprendizaje y calificación final, tal vez no disponga de suficientes elementos a la hora de medir el esfuerzo y el aprendizaje de forma individual, ni por supuesto de reflejar esas diferencias en las calificaciones individuales, que recordemos han sido bastante elevadas y homogéneas. En otras palabras, los estudiantes están satisfechos con una calificación elevada, pero algunos de ellos parecen percibir la homogeneidad en las calificaciones como un síntoma de que el sistema de evaluación no es capaz de discriminar diferentes grados de esfuerzo. Este hándicap, sin embargo, lo entendemos como inherente a la evaluación de cualquier trabajo en equipo y excede las fronteras del ámbito universitario. El trabajo cooperativo es una de las competencias profesionales más demandadas en nuestra sociedad actual y, para ese fin, es imprescindible incorporar al aula elementos de aprendizaje cooperativo que acerquen al alumno a un contexto más real.

Por último, pese a las dificultades y limitaciones descritas asociadas a esta experiencia de enseñanza-aprendizaje, el nuevo EEES nos ofrece un marco único para reflexionar sobre las posibilidades de suplir con imaginación la falta de recursos y de importar con éxito algunas características de esta experiencia. En ese ámbito pretende precisamente enmarcarse esta contribución, no en ofrecer un "manual de buenas prácticas" (lo que excede con mucho el alcance previsto de este trabajo), sino en generar y favorecer el necesario debate que debe acompañar este proceso de implantación y consolidación del nuevo EEES.

\section{Bibliografía}

Abbott, A., Butler, D., Gibney, E., Schiermeier, Q., \& Van Noorden, R. (2016). Boon or burden: What has the EU ever done for science? Nature News, 53(7607), 307-309. (DOI: https://doi.org/10.1038/534307a)

Arribas, J.M. (2012). El rendimiento académico en función del sistema de evaluación empleado. RELIEVE, Revista Electrónica de Investigación y Evaluación Educativa, 


18(1), art. 3, 1-15. (Disponible en:
https://www.uv.es/RELIEVE/v18n1/RELIEVEv18n1 3.pdf)

Aggarwall, P \& O’Brien, C.L. (2008). Social loafing on group projects: Structural antecedents and effect on student satisfaction. Journal of Marketing Education, 30(3), 255-264. (DOI: https://doi.org/10.1177/0273475308322283)

Arbizu, F., Lobato, C., \& del Castillo, L. (2005). Algunos modelos de abordaje de la tutoría universitaria. Revista de Psicodidáctica, 10(1), 7-21. (Disponible en: http://www.redalyc.org/articulo.oa?id=17514745002)

Bacon D.R., Stewart, K.A. \& Silver, W.S. (1999). Lessons from the best and worst student team experiences: How a teacher can make the difference. Journal of Management Education, 23(5), 467-488. (DOI: https://doi.org/10.1177/105256299902300503)

Bolívar, A. (2007). La planificación por competencias en la reforma de Bolonia de la Educación Superior. Un análisis crítico. Educaçao Temática Digital, 9, 68-94. (Disponible en: http://nbn-resolving.de/urn:nbn:de:0168-ssoar-73427)

Boud, D. \& Falchikov, N. (2007). Rethinking Assessment in Higher Education. Learning for the Long Term. Oxon (Reino Unido): Routledge.

Brady, L. (1985). Models and Methods of Teaching. London (Reino Unido): Prentice-Hall.

Cabrera, A.F. (2003). Evaluación de la Formación. Madrid: Síntesis.

Cohen, A.P., Kulik, J.A., \& Kulik, C.C. (1982). Educational outcomes of tutoring: A metaanalysis of findings. American Educational Research Journal, 19(2), 237-248. (Disponible en: www.jstor.org/stable/1162567)

Chapman, K.J., Meuter, M., Toy, D. \& Wright, L. (2006). Can't we pick our own groups? The influence of group selection methods on group dynamics and outcomes. Journal of Management Education, 30(4), 557-569. (DOI: https://doi.org/10.1177/1052562905284872)

Crouch, C.H. \& Mazur, E. (2001). Peer Instruction: Ten years of experience and results. American Association of Physics Teachers, 69(9), 970-977. (DOI: https://doi.org/10.1119/1.1374249) 
Cuevas-Salazar, O., García-López, R.I., Vales-García, J.J., \& Cruz-Medina, I.R. (2017). Monitoring the results of the tutoring program in its face-to-face and virtual modalities on the academic achievement of students at a Mexican University. International Journal of Higher Education, 6(2), 169-181. (DOI: https://doi.org/10.5430/ijhe.v6n2p169)

Emig, J. (1977). Writing as a mode of learning. College Composition and Communication, 28, 122-128. (DOI: https://doi.org/10.2307/356095)

Fagen, A.P., Crouch, C.H. \& Mazur, E. (2002). Peer instruction: Results from a range of classrooms. The Physics Teacher, 40(4), 2006-2009. (DOI: https://doi.org/10.1119/1.1474140)

García, A. (2009). El desarrollo de competencias apoyado en trabajo en equipo, telemática interactiva y evaluación compartida. In J.I. Aguaded, \& M.C. Fonseca (Eds), Huellas de Innovación Docente en las Aulas Universitarias. La Coruña: Netbiblo.

Hénard, F., Diamond, L. \& Roseveare, D. (2012). Approaches to Internationalisation and Their Implications for Strategic Management and Institutional Practice: A Guide for Higher Education Institutions. OECD Higher Education Programme IMHE. (Disponible en:

http://www.oecd.org/edu/imhe/Approaches\%20to\%20internationalisation\%20\%20final\%20-\%20web.pdf)

Ingham, A.G., Levinger, G., Graves, J. \& Peckham, V. (1974). The Ringelmann effect: Studies of group size and group performance. Journal of Experimental Social Psychology, 10(4), 371-384. (DOI: https://doi.org/10.1016/0022-1031(74)90033-X)

Jonassen, D.H. (1991). Objetivism vs. constructivism: Do we need a new paradigm? Educational Technology: Research and Development, 39(3), 5-14. (DOI: https://doi.org/10.1007/BF02296434)

Kafai, Y. \& Resnick, M. (Eds.) (1996). Constructivism in Practice: Designing, Thinking and Learning in a Digital World. Mahwah, NJ (Estados Unidos): Lawrence Erlbaum Associates. 
King, P.E. \& Behnke, R.R. (2005). Problems associated with evaluating student performance in groups. College Teaching, 53(2), 57-61. (DOI: http://dx.doi.org/10.3200/CTCH.53.2.57-61)

Lage, M.J., Platt, G.J. \& Treglia, M. (2000). Inverting the classroom: A gateway to creating an inclusive learning environment. The Journal of Economic Education, 31(1), 30-43. (DOI: http://dx.doi.org/10.1080/00220480009596759)

Lasnier, F. (2000). Reussir la Formation par Competences. Montreal (Canadá): Guerin.

López-Pastor, V.M. (2012). Evaluación formativa y compartida en la universidad: clarificación de conceptos y propuestas de intervención desde la Red Interuniversitaria de Evaluación Formativa. Psychology, Society, \& Education, 4(1), 117-130. (Disponible en: https://dialnet.unirioja.es/servlet/articulo?codigo=3961371)

Luján-Mora, S. (2013). De la clase magistral tradicional al MOOC: Doce años de evolución de una asignatura sobre programación de aplicaciones web. Revista de Docencia Universitaria 11, 279-300. (DOI: https://doi.org/10.4995/redu.2013.5557)

Marín-Díaz, V., Cabero-Almenara, J. \& Barroso-Osuna, J. (2012). La rúbrica de evaluación en el proceso de formación del docente universitario. La propuesta del proyecto DIPRO 2.0. Educar, 48(2), 347-364. (Disponible en: http://www.redalyc.org/articulo.oa?id=342130839009)

Márquez, J., Roca, J., Solvas, M.J., Belmonte, T., Fernández, C. \& Rodríguez, D. (2011). Resultados de aprendizaje en los nuevos títulos de grado. RED-DUSC, Revista de Educación a Distancia - Docencia Universitaria en la Sociedad del Conocimiento, 5, 1-16. (Disponible en: http://www.um.es/ead/reddusc/5/roca.pdf)

Mello, J.A. (1993). Improving individual member accountability in small work group settings. Journal of Management Education, 17(2), 253-259. (DOI: https://doi.org/10.1177/105256299301700210)

Ministerio de Educación, Cultura y Deporte (2014). Estrategia para la Internacionalización de las Universidades Españolas 2015-2020. Secretaría General Técnica, Centro de Publicaciones, Ministerio de Educación, Cultura y Deporte, Gobierno de España. (Disponible en: https://www.mecd.gob.es/educacion-mecd/dms/mecd/educacion- 
mecd/areas-educacion/universidades/politica-internacional/estrategiainternacionalizacion/Estrategialnternacionalizaci-n-Final.pdf)

Nicol, D. \& Macfarlane-Dick, D. (2006). Formative assessment and self-regulated learning: a model and seven principles of good feedback practice. Studies in Higher Education, 31(2), 199-218. (DOI: https://doi.org/10.1080/03075070600572090)

Nunes de Oliveira, J.M. (2011). Nine years of project-based learning in engineering. Revista de Docencia Universitaria, 9(1), 45-55. (DOI: https://doi.org/10.4995/redu.2011.6179)

OCDE (2012). Indicator C4 - who studies abroad and where?. Education at a Glance 2012: OECD Indicators, 360-381. (DOI: https://doi.org/10.1787/eag-2012-en)

Pfaff, E. \& Haddleston, P. (2003). Does it matter if I hate teamwork? What impacts student attitudes toward teamwork. Journal of Marketing Education, 25(1), 37-45. (DOI: https://doi.org/10.1177/0273475302250571)

Román, C., Millán, A. \& Millán, J.M. (2016). Writing to learn: An experience applied within the subject Intermediate Macroeconomics. Culture and Education, 28(2), 396-418. (DOI: https://doi.org/10.1080/11356405.2016.1158450)

Strong, J.T. \& Anderson, R.E. (1990). Free-riding in group projects: Control mechanisms and preliminary data. Journal of Marketing Education, 12(2), 61-67. (DOI: https://doi.org/10.1177/027347539001200208)

Torres-Pérez, A. (2012). Presentaciones orales en clase: Retos y perspectivas. Textos de docència Obsei, $\quad 2, \quad 51-60 . \quad$ (Disponible http://www.raco.cat/index.php/TextosObsei/article/view/251011/335892)

Weller, L.D. \& Hartley, S.H. (1994). Teamwork and cooperative learning: An educational perspective for businesses. Quality Management Journal, 1(4), 30-41. (Disponible en: http://asq.org/pub/ami/past/backissues/1994/july.html)

Weaver, M.R. (2006). Do students value feedback? Student perceptions of tutors' written responses. Assessment \& Evaluation in Higher Education, 31(3), 379-394. (DOI: https://doi.org/10.1080/02602930500353061) 
Williams, D.L., Beard, J.D. \& Rymer, J. (1991). Team projects: Achieving their full potential. Journal of Marketing Education, 13(2), 45-53. (DOI: https://doi.org/10.1177/027347539101300208)

\section{REFERENCIA BIBLIOGRÁFICA}

Millán Tapia, J.M.; Millán Tapia, A. y Román Díaz, C. (2017). Estudio de una experiencia internacional de aprendizaje interactivo en la asignatura Pequeñas Empresas e Iniciativa Empresarial. Aula de Encuentro, 19 (2), pp. 97-143. doi: https://dx.doi.org/10.17561/ae.v19i2.5

José María Millán Tapia es

Profesor Titular del Dpto. de Economía de la Universidad de Huelva Correo-e: jose.millan@dege.uhu.es

Ana Millán Tapia es Profesora Ayudante Doctora del Dpto. de Economía Financiera y Contabilidad de la Universidad Pablo de Olavide Correo-e: amillan@upo.es

Concepción Román Díaz es Profesora Contratada Doctora del Dpto. de Economía de la Universidad de Huelva Correo-e: concepcion.roman@dege.uhu.es 


\title{
NOTAS
}

\begin{abstract}
${ }^{1}$ Sería injusto decir que la situación laboral holandesa en la actualidad (con cifras de paro por debajo del 5\%) responde a un mejor funcionamiento de su sistema educativo, que es capaz de dotar a los estudiantes con la experiencia y habilidades demandados por su mercado laboral. Tan injusto, probablemente, como decir que su sistema educativo no tiene un papel relevante en esta ecuación.

2 En economía se llama "polizones" (o free-riders) a aquellos individuos o entes que consumen más que una parte equitativa de un recurso o no afrontan una parte justa del coste de su producción. En nuestro caso, el problema emerge porque no todos los estudiantes contribuyen del mismo modo a la realización del trabajo final, lo cual no es capturado por un sistema de evaluación basado mayoritariamente en el trabajo en grupo. La literatura ha analizado este fenómeno y ha ofrecido diversas soluciones. Véanse Ingham, Levinger, Graves \& Peckham, 1974; Strong \& Anderson, 1990; Williams, Beard \& Rymer, 1991; Mello, 1993; Weller \& Hartley, 1994; Bacon, Stewart \& Silver, 1999; Pfaff \& Haddleston, 2003; Chapman, Meuter, Toy \& Wright, 2006; y Aggarwall \& O'Brien 2008.

${ }^{3}$ Identificamos como "héroes" a aquellos individuos que aumentan voluntariamente su carga de trabajo para "salvar el proyecto", completando por su cuenta toda (o gran parte de) la tarea (Pfaff \& Haddleston 2003). Los "héroes" ni practican ni adquieren las habilidades sociales necesarias del trabajo en equipo, y niegan a sus compañeros la oportunidad de adquirir las habilidades técnicas que ellos ya poseen. Debido a su naturaleza perfeccionista, no están dispuestos a delegar el trabajo, ni a apreciar el trabajo realizado por otra persona. Simplemente lo rechazan y lo rehacen, desmoralizando al resto de miembros del
\end{abstract} equipo.

${ }^{4}$ Utilizando las denominaciones originales, la asignatura se llama Seminar on Small Business and Entrepreneurship y se enmarca en el programa Master's specialisation in Entrepreneurship, Strategy and Organization Economics. Este Máster fue ofertado por el Department of Applied Economics de la Erasmus School of Economics (ESE) perteneciente a la Erasmus University Rotterdam (EUR). La oferta actual de másteres internacionales ofertados por esta universidad puede encontrarse en el siguiente vínculo: <https://www.eur.nl/english/master/programmes/>.

5 De entre las cerca de 8.000 universidades en todo el mundo, la Universidad Erasmus de Rotterdam aparece de manera consistente desde el año 2003 entre las 200 mejores del Academic Ranking of World Universities -ARWU-. También conocido como el Shanghai Ranking, este índice es elaborado por la Shanghai Jiao Tong University y está considerado como el ranking de universidades más consolidado a nivel mundial. Dentro del campo de Ciencias Sociales, la Universidad Erasmus de Rotterdam aparece siempre entre las 100 primeras de este ranking, ocupando la posición 30 en el último año disponible 2016. Ya en el área de Economía y Empresa, su posición no ha bajado de las 75 primeras, ocupando la posición 29 en el último año disponible 2015. Este índice puede consultarse en el siguiente vínculo: <http://www.shanghairanking.com/>.

${ }^{6}$ Entre los graduados en estas aulas, se encuentran nombres como Neelie Kroes, que fue vicepresidenta de la Comisión Europea y comisaria de la Agenda Digital, o Jan Tinbergen, ganador del primer Premio Nobel de Economía.

${ }^{7}$ Durante el desarrollo del cuestionario que sirve de base para este trabajo, uno de los estudiantes extranjeros no contestó a la pregunta sobre el país de nacimiento. Pese a que hubiera sido posible acudir al listado oficial de alumnos matriculados para obtener ese dato, preferimos respetar la decisión del estudiante de no prestar esa información para este análisis. La sección 3 de este trabajo describe el diseño de la investigación y del cuestionario en cuestión.

${ }^{8}$ El estudiante extranjero que no contestó a la pregunta sobre el país de nacimiento también cursó sus estudios de Grado en Holanda.

${ }_{9}$ El conjunto de las instituciones extranjeras está formado por las siguientes universidades: University of Piraeus (Grecia); University of Patras (Grecia); Athens University of Economics and Business (Grecia); Riga Technical University (Letonia); Universidad de Costa Rica (Costa Rica); University of Zurich (Suiza); Universidade Federal do Rio de Janeiro (Brasil); y State University - Higher School of Economics (Rusia). Las instituciones holandesas son, por su parte, las siguientes: Erasmus University Rotterdam; Inholland University; Maastricht University; University of Groningen; The Hague University; y Avans University of Applied Science.

${ }^{10}$ Las preguntas de elección múltiple incluidas en el bloque $\mathrm{C}$ referidas a experiencia laboral permitían elegir más de una categoría, pues el mismo estudiante puede haber trabajado como empleado asalariado, ayuda familiar o emprendedor, tanto en las modalidades de tiempo parcial como completo. Por ello, el número de experiencias laborales detectadas (42) supera el número de estudiantes (24). La sección 3 de este trabajo describe el diseño de la investigación y del cuestionario.

${ }^{11}$ En el periodo $2014-20$ se estima que la UE-28 destinará 74.800 millones de $€$ a proyectos internacionales de investigación colaborativos (Abbott, Butler, Gibney, Schiermeier \& Van Noorden, 2016).

${ }^{12}$ El docente alemán es doctor por la Technical University Munich (Alemania), los doctores españoles lo son por la Universidad de Huelva mientras que los holandeses son doctores por la Erasmus University Rotterdam.

${ }_{13}$ Entre las revistas de impacto en que se encuentran las publicaciones de estos docentes, destacan las siguientes: Administrative Science Quarterly; Applied Economics; Asia Pacific Journal of Management; Economics Letters; Entrepreneurship Theory and Practice; Family Business Review; Industrial and Corporate Change; International Entrepreneurship and Management Journal; Journal of Business Ethics; Journal of Business Venturing; Journal of Economic Issues, Journal of Evolutionary Economics; Journal of Family Business Strategy; Journal of Industry, Competition and Trade; Kyklos; Research Policy; Small Business Economics; Technovation; y Venture Capital.

${ }^{14}$ En particular, se cuenta con un estudiante de doctorado que presta labor de apoyo a la docencia (teaching assistant), y que es el encargado tanto de comprobar que los trabajos se entregan a tiempo como de reenviarlos a sus destinatarios. Además, se encarga de gestionar la plataforma virtual de la asignatura (blackboard), en la que se publican los documentos de trabajo (material docente, datos, etc.), las comunicaciones o anuncios que se quieran trasladar a los estudiantes, y las calificaciones parciales y finales de la asignatura.

${ }^{15}$ Los trabajos versaban sobre innovación, exportación, contribución al empleo, satisfacción en el empleo y empleo por cuenta propia.

${ }^{16}$ En ese sentido, los estudiantes tienen una oportunidad de hacer uso de este aprendizaje en la realización del trabajo de fin de Máster en junio de 2011.

${ }_{17}$ El emprendedor en cuestión fue uno de los fundadores y propietarios de la empresa InThere - Virtual Worlds \& Gaming Solutions. Más información sobre esta empresa está disponible en http://inthere.nl/. 
18 Esta institución nace en 1930 y está integrada desde 2010 en Panteia B.V. Más información disponible en https://www.panteia.com/our-history/.

${ }^{19}$ Aunque la opinión de los estudiantes era tenida en cuenta a la hora de designar el equipo ganador, no tenía por qué haber una coincidencia final. Sin embargo, en este caso, las opiniones de estudiantes y profesores apuntaron al mismo equipo.

${ }_{20}$ En este caso, el emprendedor fue uno de los fundadores y propietarios de la empresa lcySolutions - Trust, Care \& Innovation. Más información sobre esta empresa está disponible en https://www.linkedin.com/company/icysolutions/.

${ }^{21}$ Así como en los "Trabajos de Investigación en Iniciativa Empresarial" el objetivo era aprender una metodología de trabajo e investigación, sin que el objeto de análisis fuera determinante, en el caso de los "Trabajos sobre Estrategia Empresarial", el contenido explícito de la tarea sí forma parte de los contenidos que el temario de esta asignatura pretende cubrir.

${ }^{22}$ Con 3 posibles tareas y 6 grupos de estudiantes, la distribución se realiza de forma que sean siempre 2 grupos los que se encarguen de cada una de las tareas.

${ }^{23}$ En particular, se revisa el método de Flujo de Fondos Descontados (Discounted Cash Flow Valuation), y el método de la Valoración por Múltiples Comparables (Valuation using Multiples). El uso del primer método está más extendido. Su objetivo es determinar el valor actual de los flujos de fondos futuros (cobros y pagos) descontándolos a una tasa que refleja el coste de capital aportado. Esto es necesario porque los flujos de fondos en diversos períodos no pueden ser comparados directamente puesto que no es lo mismo contar con una cantidad de dinero ahora que en el futuro. El segundo adquiere relevancia para valorar empresas que no cotizan en bolsa (para las que no existe, en consecuencia, un precio fijado en el mercado). A grandes rasgos, consiste en comparar la empresa que se quiere valorar con otras empresas o con los valores medios del sector en el que opera. Ambos métodos son conocidos por los estudiantes, pues se incluyen habitualmente en asignaturas del área de Finanzas en los estudios de Grado.

${ }^{24}$ Podría argumentarse que recibir la calificación final inmediatamente antes de cumplimentar nuestro cuestionario podría introducir algún tipo de sesgo en las respuestas de los estudiantes, en la medida en que estas calificaciones superen o no alcancen las expectativas previas. Sin embargo, las calificaciones finales apenas mostraron variaciones con respecto a las calificaciones parciales de la asignatura, correspondientes a los dos bloques previos (Investigación en Iniciativa Empresarial y Estrategia Empresarial), que ya habían sido publicadas en la plataforma virtual de la asignatura. Por otra parte, nuestro cuestionario incluye aspectos sobre satisfacción con la calificación final, por lo que era necesario ser cumplimentado conociendo esta calificación.

${ }^{25}$ En cada sesión, se entrega a cada estudiante una cartulina con su nombre que deben ubicar en lugar visible en su pupitre, lo que facilita el seguimiento de las participaciones de cada estudiante. Además, la reducida ratio alumnos-profesores permite asignar a cada profesor el control de 4 o 5 estudiantes, lo que facilita la tarea.

${ }_{27}^{26}$ El cuestionario original fue redactado en inglés y está disponible bajo petición.

${ }^{27}$ A pesar de que la escala Likert se considera generalmente una escala de tipo ordinal, ya que no podemos asumir que los sujetos perciban las respuestas como equidistantes, esta escala puede asumirse como cardinal si cada pregunta del cuestionario viene acompañada de una escala visual horizontal en la cual el entrevistado deba marcar su respuesta, y en la que cada respuesta esté situada de forma equidistante. Ese es precisamente el formato utilizado en el diseño de este cuestionario, lo que nos permite hallar e interpretar los valores medios y sus respectivas desviaciones típicas.

${ }^{28}$ Podría argumentarse que estos estudiantes muestran por lo general algunas actitudes muy positivas, tales como trabajar un mayor número de horas, o ser más exigentes consigo mismos, y con la calidad de la formación que reciben. Por el contrario, estas actitudes también coexisten con frecuencia con otras características menos deseables como el individualismo o la competitividad.

${ }^{29}$ Como advertimos en la subsección dedicada a "Resultados académicos" (Sección 2), las calificaciones finales otorgadas a los estudiantes en esta asignatura han sido bastante elevadas y homogéneas, variando entre el 6,9 y el 8,6 (en una escala de 0 a 10). Precisamente esta homogeneidad en las calificaciones, que ya íbamos anticipando en las calificaciones parciales, nos hizo decantarnos por no incluir en el cuestionario la pregunta específica sobre la calificación final obtenida en la asignatura. Así, consideramos que la información adicional relevante que hubiera podido obtenerse era reducida, en comparación con la potencial pérdida de anonimato percibida por los estudiantes en la cumplimentación del cuestionario (y a la potencial reducción en el grado de honestidad en las respuestas). 